NBER WORKING PAPER SERIES

\title{
SELF-ENFORCING STOCHASTIC MONITORING AND THE SEPARATION OF DEBT AND EQUITY CLAIMS
}

\author{
Harold L. Cole \\ Working Paper 14480 \\ http://www.nber.org/papers/w14480

\section{NATIONAL BUREAU OF ECONOMIC RESEARCH} \\ 1050 Massachusetts Avenue \\ Cambridge, MA 02138 \\ November 2008
}

I would like to thank Narayana Kocherlakota, Jon Pogach, Andy Postelwaite, Martin Schneider, Vasiliki Skreta and Xi Weng for their comments. I gratefully acknowledges support from the National Science Foundation. The views expressed herein are those of the author(s) and do not necessarily reflect the views of the National Bureau of Economic Research.

NBER working papers are circulated for discussion and comment purposes. They have not been peerreviewed or been subject to the review by the NBER Board of Directors that accompanies official NBER publications.

(C) 2008 by Harold L. Cole. All rights reserved. Short sections of text, not to exceed two paragraphs, may be quoted without explicit permission provided that full credit, including $\bigcirc$ notice, is given to the source. 
Self-Enforcing Stochastic Monitoring and the Separation of Debt and Equity Claims

Harold L. Cole

NBER Working Paper No. 14480

November 2008

JEL No. G3

\begin{abstract}
$\underline{\text { ABSTRACT }}$
This paper studies the incentive issues associated with self-enforcing stochastic monitoring in a model of investment and production. The efficient contract features a debt-like payment with a threshold in terms of the reported output in which all of the reported output is taken up to the threshold if monitoring doesn't occur and all of the output is taken if monitoring does occur. An output report above the threshold leads to zero probability of monitoring and just the threshold amount being paid out. The efficiency gap between the self-enforcing contract and the commitment constraint is minimized when the monitors hold no part of the residual claim on the firm, which we associate with equity. Misreporting by the manager is an important component of the efficient contract.
\end{abstract}

Harold L. Cole

Economics Department

University of Pennsylvania

3718 Locust Walk

160 McNeil Building

Philadelphia, PA 19104

and NBER

colehl@sas.upenn.edu 


\section{Introduction}

This paper studies the incentive issues associated with stochastic monitoring in a model of investment and production. In the model outside investors hire a manager in order to run a project and produce output. The output of this project is subject to a short-run information friction and a longer-run agency friction. The agency friction motivates the outside investors to try and extract their funds early, while the information friction makes it easier to extract their funds later. The optimal extraction process is determined by a trade-off between these two factors. Just as in Townsend (1979), monitoring can be used to overcome the information friction, both because it allows the investors to see the actual output when they monitor and to encourage more accurate reporting when they don't. However, monitoring is ex post inefficient since it involves dead weight losses. This ex post inefficiency makes it difficult to generate the right incentives with respect to the monitors when we require it to be self-enforcing.

The analysis of the impact of self-enforcing monitoring builds off of a recent paper by Atkeson and Cole (2005) (AC). They showed that a model with a temporary information friction, an agency friction and deterministic monitoring could generate two distinct payment streams within each period, an interim debt-like payment and a residual equity-like payment, along with a theory of executive compensation. But, like many papers in the literature, AC left unanswered the question of which payments should and should not be bundled together and the extent to which their results depended upon deterministic monitoring. ${ }^{1}$ Here we show that efficiently generating the correct monitoring incentives motivates the separation of debt and equity claims in order to restrict the monitors to only hold debt claims. We also show that AC's results carry over to a stochastic monitoring context.

For monitoring to be self-enforcing, the monitors must obtain a financial benefit from monitoring which covers their costs. This necessitates a wedge between the interim or debt payout when monitoring occurs and when it does not occur. We show that it's easy to construct a self-enforcing contract that supports the efficient outcome obtained under commitment when monitoring is deterministic and the expected value of the debt payment is big enough. However, when the debt payment is not big enough additional costly liquid funds have to be invested into the firm in order to compensate the monitors at the commitment level of debt. This introduces a trade-off into the model between the size of the debt and the amount of liquid funds that must be invested into the firm. Since increasing the size of

\footnotetext{
${ }^{1}$ DeMarzo and Fishman (2003) also construct a model in which the optimal contract can be split into three pieces, outside debt, outside equity, and a credit line. Here too there is no fundamental reason for this division.
} 
the debt means increasing the extent of monitoring, both adjustments are costly. We show that having the monitors only hold debt claims on the firm reduces the minimum debt level below which costly funds must be invested while also reducing the extent to which costly liquid funds need to be invested when the level of debt is below this threshold.

We allow for stochastic monitoring because it can be used to minimize the extent of monitoring sufficient to generate the correct incentives with respect to managerial reporting. Stochastic monitoring exacerbates the incentive problem of the monitors relative to deterministic monitoring because the probability with which the monitors are suppose to monitor conveys information about the state of the firm. This tightening of the incentive friction for the monitors means that the efficient stochastic monitoring contract with commitment is not self-enforcing. We characterize the self-enforcing stochastic monitoring contract and show that despite allowing for stochastic monitoring and the additional incentive constraint that monitoring be self-enforcing, the general nature of the results in AC go through. There still is a debt-like payment with a threshold in terms of the reported output in which all of the reported output is taken up to the threshold if monitoring doesn't occur and all of the output is taken if monitoring does occur. An output report above the threshold leads to zero probability of monitoring and just the threshold amount being paid out. Just as with deterministic monitoring, we find that the efficiency gap between the self-enforcing contract and the commitment constraint is minimized when the monitors holds no part of the residual claim on the firm, which we associate with equity. Hence, the bundling together of the payouts that we associate with debt and equity is inefficient in this model, and our results provide an answer for separating the payouts of the firm into debt and equity.

Our results on the efficiency of multiple claims on the firm is similar in spirit to findings of Dewatripont and Tirole (1994) and Berglof and von Thadden (1994) who find that there is an optimal distribution of short-term vs. long-term claims within models of complete but noncontractible information. In their models short-term claims are better able to insist on liquidation in bad intermediate output states or if short-term payments are not made because they have stronger bargaining position ex post. They find that the right combination of short-term and longer-term claims helps to generate the appropriate choice of firm liquidation, which in turn generates the correct incentives for debt payments. Our result is complementary to theirs in that it shows that getting monitoring incentives right generates a more extreme prediction that the monitors should only hold debt claims on the firm. ${ }^{2}$

\footnotetext{
${ }^{2}$ Maskin and Tirole (1999) argues that the noncontractible information assumption is problematic since there are simple games that can in effect elicit this information. This assumption is particularly contentious in this context since one could offer the debt holders the option of a long-term claim on the firm if their short-term claim was not paid off. If they can sell this claim at a value that reflects the true state of the world, then this elicits the noncontractible information.
} 
There is another important difference between the contract with self-enforcing monitoring relative to complete enforcement. First, misreporting of output is now an important element in supporting the efficient outcome, and occurs with positive probability. It is these misreports which generate the financial gains to the monitors from monitoring, and hence leads them to have an incentive to monitor. In equilibrium there are sanctioned misreports which are being used to satisfy the incentive constraint of the monitors, and nonsanctioned misreports. Sanctioned misreports, which are suppose to occur in equilibrium, are not punished in an ex ante sense since the manager must be indifferent between truthtelling and misreporting in equilibrium. Nonsanctioned misreports on the other hand must be punished. These predictions with respect to the frequency and treatment of misreporting are loosely consistent with the empirical evidence. Kedia and Philippon (2006) document that many firms have had to revise their financial statements. These restatements can be extremely large, as in the well known case of Enron. While very large restatements, such as in case of Enron, do involve strong sanctions against the firm's management, small misreports seems to involve little or no punishment for management.

This paper is related to related to a wide literature on the optimal financial contract between outside investors and a manager in the presence of both information and agency frictions when there is the possibility of monitoring. The model considered here is most closely related to AC. Aspects of the information and monitoring components are similar to Townsend (1979) and Gale and Hellwig (1985), ${ }^{3}$ and aspects of the agency friction and the information friction are similar to Hart and Moore (1995). ${ }^{4}$ Also as in AC, the inclusion of both frictions and monitoring, and the specific form of these friction leads to three different payment streams coming out of the firm, outside debt, outside equity, and managerial compensation. However, unlike AC, our model provides a rationale for why the outside claims cannot be combined into one.

This paper is also related to the literature on optimal stochastic monitoring and optimal self-enforcing monitoring. Mookherjee and Png (1989), and Border and Sobel (1987) characterize efficient stochastic auditing models with commitment. ${ }^{5}$ An important difference between their models and this one is that in the absence of monitoring, the principal never sees the output level of the agent. Hence, the information problem is persistent, and

\footnotetext{
${ }^{3}$ Also related are Wang's (2004) dynamic costly-state-verification model and Williamson's (1987) model of monitoring with investment indivisibilities and equilibrium credit rationing.

${ }^{4}$ As in Jenson (1986) debt acts as a means of avoiding the agency friction associated with leaving funds in the firm and awaiting their payout as dividends.

${ }^{5}$ Other examples include Monnet and Quintin (2005), who consider a repeated version of Border and Sobel's model. Aiyagari and Alvarez (1995) considers the long-run implications of monitoring in a model of stochastic income.
} 
not transient as the one we study here. This leads to substantial differences in the models predictions on optimal compensation. Khalil (1997) considers a model in which there is production and stochastic auditing. He shows that the lack of commitment leads to the increased likelihood of an audit occurring.

\section{Model}

There is a collection of risk neutral outside investors who are endowed with a production technology that transforms the labor of a manager into output. There are a large number of identical risk averse managers. These managers have an outside opportunity that offers them utility $U_{0}$. The investors hire a single manager in order to run their project and promise him at least his ex ante opportunity cost.

The production process takes place over the course of three sub-periods within the period. In the first sub-period, a manager is chosen to operate the production technology. The contract being entered into between the investors and the manager is also determined at this point in time and any funds to be invested in the firm are put in. We will assume that funds invested between the first and third subperiods earn the market rate of return, but that liquid funds, which can be withdrawn in the second subperiod, earn a lower rate of return due to their higher liquidity. The per unit net cost of liquid funds invested in the firm is $\beta$.

In the second sub-period, the production technology yields stochastic output $\theta$ which is temporarily private information to the manager. The outside investors can ask the manager for a report as to the value of output and then choose whether or not to monitor the output of the project to learn the realization of $\theta$. This monitoring comes at the cost of $\gamma$ units of output. After making the monitoring decision, the investors can require an interim payment from the manager. The advantage of monitoring is that they can take all of the output of the firm, while if they don't monitor they can only take what the manager says is the output of the firm. We will allow the monitoring decision, conditional on the reported output of the firm, to be stochastic. We will assume that any report made by the manager is public information. We will also assume that any output not paid out in the second subperiod is reinvested in the firm.

In the third subperiod, the outside investors can freely observe the output of the firm in the second subperiod $\theta$. However, the manager can threaten to quit at this point. If the manager quits, the value of the resources invested in the firm, which here is simply the carried over output plus the liquid funds from the previous subperiod, will fall by the factor 
$\tau{ }^{6}$ This fall represents the loss that occurs because they didn't benefit from the manager's input. Long-term financial assets of the firm don't require the manager's input and aren't effected by his quitting. The manager can use the threat of quitting to try to renegotiate his salary. At the end of the third subperiod, all remaining output and funds, after compensating the manager, are paid to the outside investors, and consumption for both the manager and the outside investors takes place.

\section{Timing within the Period}

\begin{tabular}{|l||l||l|}
\hline Subperiod 1 & Subperiod 2 & Subperiod 3 \\
\hline i) hire the manager & i) $\theta$ realized & i) $\theta$ public \\
ii) contract & ii) manager reports & ii) renegotiate ? \\
iii) invest & iii) monitor or not & iii) pay manager \\
& iv) interim payment & iv) residual paid out \\
\hline
\end{tabular}

The set of possible output realizations, $\Theta$, is discrete, includes 0 as its lower support and has a finite upper support. The distribution of these shocks has c.d.f. $P$ with density $p$ and an expected value of one. The outside investors are risk neutral, while the manager is risk averse with preferences given by $E\{u(c)\}$ where $u^{\prime}(0)=\infty$ and $u(0)=0$.

Enforcement: We will assume that the enforcement technology with respect to contracts is limited in ways that are designed to mimic keys aspects of actual contract enforcement. First, contracts can always be renegotiated if both parties agreed to the new contract. Second, the manager can always quit. Third, none of the parties can be forced to put in additional funds, so either the parties have an incentive to make these payments, or the all funds have to invested up front. ${ }^{7}$

Wage Renegotiation: These limitations on the enforcement technology can be used by the manager to demand higher compensation. The outside investors cannot commit to not renegotiating and the manager cannot commit to not quit. Hence, the manager can use the threaten of quitting to renegotiate his wage and thereby extract some part of reduction in value that his quitting will cause. For simplicity, we will assume that he has all the bargaining power in this renegotiation and can extract the full value of the loss associated with his quitting. The manager will find it optimal to do this whenever his promised wages, which are paid at the end of the third subperiod, are below the level he can extract through

\footnotetext{
${ }^{6}$ The assumption that the liquid funds are subject to this fall in value turns out to be essentially equivalent to assuming that the manager has control over which funds are used to pay the interim payment - either liquid funds or output. See the discussion in footnote 6 and footnote 9.

${ }^{7}$ One reason for this last requirement is that claims need to be transferable and can always be transfered to an agent with limited liability who can claim to have zero wealth.
} 
renegotiating his contract. The possibility of this extraction gives the manager an incentive to misreport downward the realized output of the firm in the second subperiod in order to maximize the amount of resources invested in the firm. The threat of this extraction by the manager provides a reason for the outside investors to monitor in the second subperiod and thereby reduce the extent of expropriable resources left in the firm.

Monitoring: Monitoring is being done here to affect the reporting decision of the manager. Because the monitors can always claim a lack of funds to pay the fixed cost of monitoring, they must have an incentive to monitor. This incentive to monitor comes from the gap in their expected receipts if they monitor, relative to their receipts if they don't. As we will discuss in more detail below, the allocation of the claims for the two payments coming out of the firm - the interim payment in the second subperiod and the residual payment in the third subperiod - will affect the nature of this incentive issue for the monitors. We will show that stochastic monitoring is generally efficient relative to deterministic monitoring. However, since any reports by the manager are public and it is difficult to observe the randomization by the monitors, we will assume that they have to be precisely indifferent between monitoring and not for all interior randomizations. This requirement will make satisfying the incentive constraint of the monitors nontrivial.

\subsubsection{Contracting}

Contracting between the outside investors and the manager is complicated by the fact that both parties have incentive issues. Because the outside investors in their role as monitors also have an incentive constraint, the standard revelation principal argument does not apply to this environment. We will therefore make use of the results in Besster and Strausez (2001) (BS), who extend the revelation principal to environments in which the principal cannot fully commit to construct the contracting problem of the monitors. BS show that the optimal outcome can be supported by a direct mechanism in which truthful revelation is an optimal response but not necessarily the only equilibrium response that occurs with positive probability. Nontruthful responses are part of the optimal arrangement as they help to satisfy the incentive constraint of the principal (here the investors/monitors).

It is key to note here that BS's result is not that all of the outcomes from an arbitrary mechanism can be supported, but rather to show that all of the payoffs on the frontier of the Pareto frontier can be supported. This lack of full equivalence will lead us to construct an alternative mechanism which supports the same payoffs in order to generate a "nice" contract.

When we allow the outside investors to commit to all aspects of the contract, in particular 
the monitoring probability, the revelation principal applies to our environment. Hence, in the commitment case, we will restrict attention to the truth-telling equilibrium of a direct mechanism.

In determining the efficient contract, we will treat the investors/monitors as the principal. The contract between the outside investors and the manager can be described as follows. A contract between these parties specifies an amount $\delta$ to be invested up front, a probability of monitoring by the outside investors $m$, a payment from the manager to the outside investors in the second sub-period $v$, and a payment $x$ to the manager in the third sub-period.

The monitoring probability is a function of the manager's announcement $\hat{\theta} \in \Theta$ of the output of the project in the second sub-period, and we denote it by $m(\hat{\theta})$. The payments $v$ from the manager to the outside investors in the second sub-period are contingent on the manager's announcement of the output level $\hat{\theta}$ as well as the outcome of the monitoring decision. Let $v_{0}(\hat{\theta})$ denote the payment that the manager makes to the outside investors in the second sub-period as a function of the announcement $\hat{\theta}$ in case monitoring does not take place, and let $v_{1}(\hat{\theta}, \theta)$ denote the payment that the manager makes as a function both of the announcement $\hat{\theta}$ and the true value of $\theta$ in case monitoring does take place. Finally, let $x_{i}(\hat{\theta}, \theta)$ denote the payment from the outside investors to the manager in the third sub-period as a function of his report $\hat{\theta}$ in the second sub-period and the realized output $\theta$, where $i=0$ denotes the case in which monitoring did not take place and $i=1$ denotes that in which it did. A reporting strategy for the manager $r$ is a probability distribution over $\Theta$ conditional on the realized output level $\theta ; r(\hat{\theta}, \theta) \in \triangle(\Theta) \times \Theta \cdot r(\hat{\theta}, \theta)$ is the probability that a manager of type $\theta$ makes a report $\hat{\theta}$.

The restriction that all funds paid out either come from funds invested up front, or output leads to the following restrictions on the contract. The liquid funds invested up front must be positive, or

$$
\delta \geq 0
$$

The interim payments cannot exceed the total reported resources if monitoring doesn't take place and the total actual resources if it does, hence

$$
\begin{gathered}
v_{0}(\hat{\theta}) \leq \hat{\theta}+\delta, \\
v_{1}(\hat{\theta}, \theta) \leq \theta+\delta .
\end{gathered}
$$

The wage payment to the manager cannot be negative

$$
x_{i}(\hat{\theta}, \theta) \geq 0, \text { for } i=0,1 \text {. }
$$


The manager cannot claim to have an output level which would require him to payout more than he has available if monitoring doesn't occur, or

$$
\left[\theta+\delta-v_{0}(\hat{\theta})\right] r(\hat{\theta}, \theta) \geq 0
$$

We can think of this last restriction as arising from the fact that he must demonstrate that he has the funds to make the reported payment. Restrictions (2-5) are assumed to hold for all $\hat{\theta}, \theta \in \Theta$.

We will assume, without loss of generality, that $x_{i}(\hat{\theta}, \theta)$ is chosen to be renegotiation proof. $^{8}$ This assumption implies a constraint on $x_{0}(\hat{\theta}, \theta)$ and $x_{1}(\hat{\theta}, \theta)$ that $^{9}$

$$
\begin{aligned}
& x_{0}(\hat{\theta}, \theta) \geq \tau\left(\theta+\delta-v_{0}(\hat{\theta})\right) \text { and } \\
& x_{1}(\hat{\theta}, \theta) \geq \tau\left(\theta+\delta-v_{1}(\hat{\theta}, \theta)\right) \text { for all } \hat{\theta}, \theta .
\end{aligned}
$$

With respect to the incentive constraints on the manager, we will require that truthtelling is a best response

$$
\begin{aligned}
& m(\theta) u\left(x_{1}(\theta, \theta)\right)+(1-m(\theta)) u\left(x_{0}(\theta, \theta)\right) \\
\geq & m(\hat{\theta}) u\left(x_{1}(\hat{\theta}, \theta)\right)+(1-m(\hat{\theta})) u\left(x_{0}(\hat{\theta}, \theta)\right) \text { for all } \hat{\theta}, \theta \in \Theta .
\end{aligned}
$$

We will also require that all reports given with positive probability do as well as truth-telling

$$
\begin{aligned}
0= & {\left[\begin{array}{c}
m(\hat{\theta}) u\left(x_{1}(\hat{\theta}, \theta)\right)+(1-m(\hat{\theta})) u\left(x_{0}(\hat{\theta}, \theta)\right) \\
-m(\theta) u\left(x_{1}(\theta, \theta)\right)+(1-m(\theta)) u\left(x_{0}(\theta, \theta)\right)
\end{array}\right] r(\hat{\theta}, \theta) } \\
& \text { for all } \hat{\theta}, \theta \in \Theta .
\end{aligned}
$$

The long-term funds invested in the firm are implicitly given by the maximum of

$$
x_{i}(\hat{\theta}, \theta)-\theta-v_{i}(\hat{\theta}, \theta)
$$

\footnotetext{
${ }^{8}$ This constraint is closely related to the no-perks constraint in AC. Their constraint was motivated by the notion that managers could misuse funds for their private benefit (perks), and the no-perks constraint was imposed to ensure that they did not do so.

${ }^{9}$ If we had assumed instead that liquid funds were not subject to lossing the fraction $\tau$ of their value, but that instead the interim payment was made first with liquid funds $\delta$ (as the manager would prefer), then the condition would be $x_{i} \geq \tau \min \left\{\left[\theta-\left(v_{i}-\delta\right)\right],\left[\theta-v_{i}\right]\right\}$. This differs from the condition we impose only in that the amount that the manager can extract has an upper bound. Alternative if the output of the firm was used first, then the appropriate condition, $x_{i} \geq \tau\left(\theta-v_{i}\right)$, would be even less stringent. However, imposing either of these alternative conditions does not substantively change the qualitative results.
} 
where this maximum is over $i=0,1, \theta$ and reports $\hat{\theta}$. Since these funds are assumed to have zero net cost, their magnitude does not impact on the contracting problem. The liquid funds $\delta$ invested in the firm are costly and the only reason for investing in this form is to raise the interim payments high enough for low output levels to motivate monitoring

\subsubsection{Monitoring Incentives}

To make monitoring self-enforcing, it is necessary that the net expected payment be equal to zero if $m(\hat{\theta}) \in(0,1)$, nonnegative if $m(\hat{\theta})=1$ and nonpositive if $m(\hat{\theta})=0$. However, the net expected gain depends upon how the claims to output are distributed among the outside investors. In particular, it matters whether or not the second sub-period and the third sub-period claims can be thought of as being held by one joint investor, or by two separate investors each holding only one of these claims (and of course all the convex combinations in between). To see this why this is the case, consider the net payout in two extreme cases in which (i) an investor held all of the claims and (ii) one investor (the monitor) held the claims to second sub-period payouts and a second investor held the claims to third sub-period payouts:

1. Unseparated Claims Condition: The expected gain to the monitors is

$$
\left[E\left\{v_{1}(\hat{\theta}, \theta)\right\}-v_{0}(\hat{\theta})\right] \tau-\gamma
$$

since the agency cost will lose them at most only the fraction $\tau$ of what is not paid out in the second sub-period.

2. Separated Claims Condition: The expected gain to the monitors is

$$
\left[E\left\{v_{1}(\hat{\theta}, \theta)\right\}-v_{0}(\hat{\theta})\right]-\gamma
$$

since now the holders to the claim on the second sub-period payment will lose the full amount of anything they don't collect in this period.

The allocation of claims and the decision making process for the monitoring will determine the nature of the expectation condition that prevails. For example, if the monitors held the fraction $\rho$ of debt claims and $1-\rho$ of equity claims in his portfolio, selling the fraction $\rho$ of equity claims to other passive outside investors, then their expected gain from monitoring would be

$$
\left[E\left\{v_{1}(\hat{\theta}, \theta)\right\}-v_{0}(\hat{\theta})\right][\rho+(1-\rho) \tau]-\gamma
$$


assuming that the agency friction binds. By varying $\rho$ between 0 and 1 , one can generate an expected gain anywhere between the two extremes of unseparated and separated claims. In what follows, we will restrict attention to simple conditions of this form. ${ }^{10}$

We treat a contract as being self-enforcing if there exists a $\phi \in[\gamma, \gamma / \tau]$ such that for all $\hat{\theta} \in \Theta$, the expected differential between monitoring and not monitoring is (i) equal to $\phi$ if the monitoring probability is interior or the nonmonitoring payment is positive, (ii) greater than or equal to $\phi$ if it is occurring with probability one, and (iii) the expected differential is less than or equal to $\phi$ when monitoring is taking place with probability 0 and the nonmonitoring payment is equal to zero. Formally, the incentive conditions for the monitors is

$$
\begin{aligned}
& \sum_{\theta}\left\{\left[v_{1}(\hat{\theta}, \theta)-v_{0}(\hat{\theta})\right]-\phi\right\} r(\hat{\theta}, \theta) p(\theta)= 0 \text { if } m(\hat{\theta})=1 \\
& \text { if } m(\hat{\theta}) \in(0,1) \\
& \leq 0 \text { if } m(\hat{\theta})=0
\end{aligned}
$$

where $\phi \in[\gamma, \gamma / \tau]$, for any $\hat{\theta}$ for which reports occur with positive probability. We will show below that it is always weakly efficient to have separated claims since it implies that the incentive constraint on the monitoring is less binding on the efficient contract. ${ }^{11}$

Note that we are free to assign beliefs to induce monitoring for any reports which occur with zero probability. This is because it is possible to construct contracts which are arbitrarily close in terms of payoffs in which these beliefs are pinned down by the strategy of the manager. We discuss this briefly below.

\footnotetext{
${ }^{10}$ With completely unseperated claims, if the agency friction does not bind then there would be no gap for which the loss was equal to $\gamma$. With completely seperated claims, it does not matter whether the agency friction binds, since the residual payment is not going to the monitor. For interior $\rho$, if the agency friction doesn't bind, then the gap is

$$
E\left\{v_{1}(\hat{\theta}, \theta)\right\}-v_{0}(\hat{\theta}) \rho-\gamma,
$$

which would lead to a stronger condition. However, both this version and (9) coincide for $\rho=1$. Since we show that complete seperation is always efficient even under this loose interpretation of self-enforcement, it follows that it would hold under the alternative stricter interpretation discussed here.

${ }^{11}$ After the announcement of $\hat{\theta}$, the outside investors cannot renegotiate the nonmonitoring payment $v_{0}$ and the workers contracted wage. We rationalize this by noting that unlike the wage renegotiation that occurs in the third subperiod, this renegotiation is taking place in the second subperiod under incomplete information. Hence, the beliefs of the negotiating parties can respond to the fact that an offer to renegotiate is made, and since such an offer is an out of equilibrium move, their beliefs are not pinned down by the equilibrium of the model. It is therefore easy to construct beliefs such that an offer to renegotiate at this stage will never be fruitful.

For example, continue to assume that the manager has all the negotiating power, and hence the outside investors have no incentive to try and renegotiate. Then note that if the beliefs of the outside investors about $\theta$ respond to an offer to renegotiate on the part of the manager by increasing by more than $\phi$ plus the offer, then the offer will always make them want to monitor for sure.
} 


\subsubsection{The Efficient Contract}

Taking $\phi$ as given, the problem of determining the efficient contract can be written as choos$\operatorname{ing} \delta, m(\theta), v_{0}(\hat{\theta}), v_{1}(\hat{\theta}, \theta)$ and $x_{i}(\hat{\theta}, \theta)$ so as to

$$
\max \sum_{\theta \in \Theta} \sum_{\hat{\theta} \in \Theta}\left\{\begin{array}{c}
\theta-m(\hat{\theta})\left[x_{1}(\hat{\theta}, \theta)+\gamma\right] \\
-(1-m(\hat{\theta})) x_{0}(\hat{\theta}, \theta)
\end{array}\right\} r(\hat{\theta}, \theta) p(\theta)-\beta \delta
$$

subject to the feasibility conditions (1-5), the renegotiation proof constraint on $x_{i}$, (6), the incentive constraint that truth-telling is a best response, (7), the best response constraint on other reports given with positive probability, (8), the monitoring incentive constraint (10), and finally the participation condition of the manager

$$
\sum_{\theta \in \Theta} \sum_{\hat{\theta} \in \Theta}\left[m(\hat{\theta}) u\left(x_{1}(\hat{\theta}, \theta)\right)+(1-m(\hat{\theta})) u\left(x_{0}(\hat{\theta}, \theta)\right)\right] r(\hat{\theta}, \theta) p(\theta) \geq U_{0} .
$$

This is a complicated problem, so we will attack it in stages. First, we will restrict ourselves to deterministic monitoring and show that under certain conditions the efficient solution assuming that the outside investors can commit with respect to monitoring can be supported as a self-enforcing contract. Hence, the deterministic case generates the same sort of debt and equity structure as in AC. Then we will consider the efficient contract with stochastic monitoring; first with and then without commitment. We will show that the commitment contract can not generally be supported when we impose the incentive constraint on the monitors. In addition, we will show that the efficient contract with stochastic monitoring can be mapped into an equivalent contract that looks very much like a standard debt and equity claims on the firm.

\subsection{Deterministic Monitoring}

Deterministic monitoring is a useful benchmark both because it has typically been assumed within the literature and it is simpler. With deterministic monitoring we are simply adding the requirement that $m(\theta) \in\{0,1\}$ to our programming problem. We start first with the case in which the outside investors can commit.

\subsubsection{Commitment}

If we assume that the monitors can commit with respect to their monitoring decision, we are dropping the enforcement constraint (10). Since in this case the standard revelation 
principal applies, we can restrict ourselves to a truthtelling equilibrium in which $r(\theta, \theta)=1$ and $r(\hat{\theta}, \theta)=0$ for all $\hat{\theta} \neq \theta$. In addition, since inclusion of costly liquid funds $\delta$ is to help motivate the monitors to monitor, it follows that with commitment $\delta=0$, and the problem collapses down to being a simplified version of the contracting problem considered in AC. They showed that the efficient contract had the following properties (except for sixth property since they didn't include $\delta$ ).

Proposition 2.1. There is an efficient contract with the following properties:

1. $v_{1}(\hat{\theta}, \theta)=\theta$ for all $\hat{\theta} \in\{\hat{\theta} \in \Theta: m(\hat{\theta})=1\}$,

2. $v_{0}(\hat{\theta})=\theta^{*}$ for all $\hat{\theta} \in\{\hat{\theta} \in \Theta: m(\hat{\theta})=0\}$, where $\theta^{*}=\min \{\hat{\theta} \mid m(\hat{\theta})=0\}$,

3. $m(\hat{\theta})=1$ for all $\hat{\theta}<\theta^{*}$,

4. for $\hat{\theta} \neq \theta, x_{1}(\hat{\theta}, \theta)=0$ if $\hat{\theta}<\theta^{*}$ and $x_{0}(\hat{\theta}, \theta)=\tau\left(\theta-\theta^{*}\right)$ if $\theta>\theta^{*}$,

5. the equilibrium payments to the manager have the form $x_{i}(\theta, \theta)=w(\theta)$ for $i=1,2$, where $w(\theta)=\max \{\bar{w}, \tau(\theta-\theta)\}$, and

6. $\delta=0$.

Proof: See Atkeson and Cole (2005).

In their proof, AC noted that condition (1) and (4) made the punishments as large as possible given (2). They then noted that given (4), one never wanted to tell a lie that lead to monitoring, and that that making $v_{0}(\hat{\theta})$ as large as possible weakly relaxed the renegotiation proofness (their no-perks) constraint. However, since $\min \left\{v_{0}(\hat{\theta}): \hat{\theta} \in \Theta\right.$ and $\left.m(\hat{\theta})=0\right\} \leq$ $\theta^{*}$, it followed that the best misreport would be $\theta^{*}$ and this potential misreport would determine the extent to which the no-perks constraint bound in equilibrium. Therefore, there was no gain to raising $v_{0}(\theta)$ above $\theta^{*}$, hence (2) follows. Then they noted that any monitoring above $\theta^{*}$ did not relax the incentive constraint and hence (3) follows. Given (1) - (4), it follows that the renegotiation constraint and the incentive constraint reduce to the requirement that

$$
u(w(\theta)) \geq u\left(\tau\left(\theta-\theta^{*}\right)\right) \text { for all } \theta \geq \theta^{*} .
$$

Since the manager is risk averse, it follows that compensation should be constant unless constraint (13) binds, and this implies (5).

This characterization implies that the interim payment $v_{0}(\hat{\theta})$ looks like a debt contract with the amount owed being $\theta^{*}$, and the failure to pay $\theta^{*}$ leading to monitoring and holders 
of the claim to $v_{0}(\hat{\theta})$ receiving the output of the firm up to $\theta^{*}$. Optimal compensation comes in the form of a base wage $\bar{w}$ and a performance bonus $w(\theta)-\bar{w}$ triggered by a high output or profit level of the firm. Since $\bar{w}$ must be paid even if the output of the firm is zero, we will assume that $\bar{w}$ is invested up-front in long-term funds rather than collected at the end from the outside investors. Given this, the claim to the residual payment in the third period resembles equity: it pays out 0 if $\theta<\theta^{*}$, and $\theta-\theta^{*}-(w(\theta)-\bar{w})$ for $\theta \geq \theta^{*}$, which is increasing in $\theta$. AC show that these features emerge in a richer dynamic model.

\subsubsection{Without Commitment}

Here we consider trying to construct a self-enforcing contract that will be able to support the commitment outcome under deterministic monitoring. The key to this construction is to have the manager not give a distinct report for $\theta<\theta^{*}$, and hence allow the monitors to know how much there is to be recovered if monitoring doesn't occur. The other element in altering the original contract is not to punish appropriate misreporting. If in the efficient contract (with commitment)

$$
E\left\{\theta \mid \theta<\theta^{*}\right\} \geq \phi
$$

then we will be able to completely replicate the commitment contract. If however the inequality (14) does not hold, we will need to put in costly liquid funds $\delta$ into the project or raise $\theta^{*}$. This means that if (14) does not hold the self-enforcing version of this contract will have a lower payoff than the commitment version. The additional investment $\delta$ (or the required change in $\theta^{*}$ ) is made as small as possible by setting $\phi$ to it's lowest possible value, $\gamma$, and this is achieved by having complete separation of claims in which the monitors have implicitly sold off all of their claims to the third subperiod payment.

If condition (14) holds, then the efficient contract with commitment self-enforcing takes the following form:

1. $r(0, \theta)=1$ if $\theta<\theta^{*}$ and 0 o.w.,

2. $x_{1}(\hat{\theta}, \theta)=\bar{w}$ if $\hat{\theta}<\theta^{*}$ and $\theta<\theta^{*}$, and $x_{0}(\hat{\theta}, \theta)=\max \left(\bar{w}, \tau\left(\hat{\theta}-\theta^{*}\right)\right)$

3. $\delta=0$.

4. $v_{1}(\hat{\theta}, \theta)=\theta$ if $\hat{\theta}<\theta^{*}$ and $v_{1}(\hat{\theta}, \theta)=\theta^{*}$ o.w.

$$
v_{0}(\hat{\theta})=\left\{\begin{array}{c}
\hat{\theta} \text { if } \hat{\theta} \leq \theta^{*} \\
\theta^{*} \text { o.w. }
\end{array}\right.
$$


To understand this contract, note that (1) is asking the manager to report 0 if $\theta<\theta^{*}$, and (2) is promising him the same compensation that he received under the commitment contract if he does so. Since the manager is being treated the same for any report $\hat{\theta}<\theta^{*}$ (when $\theta<\theta^{*}$ ), he is indifferent over these reports. If $\theta \geq \theta^{*}$ note that the manager does at least weakly better by telling the truth. Hence the suggested reporting strategy is a best response for the manager.

To understand why the monitors have the correct incentives, note that the monitors only know that $\theta<\theta^{*}$ when they receive a report of 0 . Hence, their expect return from monitoring is $E\left\{\theta \mid \theta<\theta^{*}\right\} \geq \phi$. For reports $\hat{\theta} \in\left(0, \theta^{*}\right)$, which occur with probability 0 , the expected return of the monitors is not pinned down by the actions of the manager and we are free to set their expectation equal to $\phi$ if they receive such a report. Given this, the monitors at least weakly prefer to monitor for any report $\hat{\theta}<\theta^{*}$. Since the expected payment to debt is $\theta^{*}$ regardless of whether or not monitoring takes place for any truthful report $\theta \geq \theta^{*}$, the monitors strictly prefer not to monitor for reports $\hat{\theta} \geq \theta^{*}$. This establishes that monitoring is self-enforcing.

Misreporting by the manager is an important component in achieving the efficient outcome. If all managers were compelled to truthfully report their output levels, then no monitoring for output levels below $\phi$ could be supported. But in this case, the best lie would always be to report 0 , and hence there would be no gain from monitoring, and monitoring would be set to 0 . This zero-monitoring outcome is only avoided because of the misreporting of the manager.

When condition (14) doesn't hold, then $\delta$ will need to be positive in order to generate the same outcome (modulo the cost of these funds $\beta \delta$ ). This will induce a trade-off between monitoring more (i.e. raising $\theta^{*}$ ) and making $\delta$ positive. Taking $\theta^{*}$ as given, we need to make the following changes to the contract relative to that when (14) holds:

$$
\begin{aligned}
& \text { 3'. } \delta=\max \left[\phi-E\left\{\theta \mid \theta<\theta^{*}\right\}, 0\right] \\
& \begin{aligned}
\text { 4'. } v_{1}(\hat{\theta}, \theta)=\theta+\delta \text { if } \hat{\theta}<\theta^{*} \text { and } v_{1}(\hat{\theta}, \theta) & =\theta^{*}+\delta \text { o.w.and } \\
v_{0}(\hat{\theta}) & =\left\{\begin{array}{l}
\hat{\theta} \text { if } \hat{\theta}<\theta^{*} \\
\theta^{*}+\delta \text { o.w. }
\end{array}\right.
\end{aligned}
\end{aligned}
$$

Note that when $\hat{\theta}<\theta^{*}$, monitoring occurs and everything is paid out, and when $\hat{\theta} \geq \theta^{*}$, monitoring does not take place and $\theta^{*}+\delta$ is paid out. Hence in equilibrium the amount left in the firm when monitoring does not occur is given by $\theta-\theta^{*}$. This in turn implies that putting in the liquid funds never effects the manager's ability to renegotiate his wage. Thus, 
the only cost of these liquid funds is their lower return. This will not be true when we turn to stochastic monitoring because the nonmonitoring payment of $\hat{\theta}$ will be occurring with positive probability for $\hat{\theta}<\theta^{*}$.

When condition (14) doesn't hold and the manager reports $\hat{\theta}<\theta^{*}$, the debt contract is no longer taking everything that the manager says he has in order to generate a larger gap between the monitoring and no monitoring payments via the additional funds $\delta$. However, by expanding the set of possible reports, one can restore this property. Consider the following contract in which $\Theta$ is expanded to include $-\delta$. With this expansion, we have the manager report $\hat{\theta}=-\delta$ if $\theta<\theta^{*}$, and we can set $v_{0}(\hat{\theta})=\hat{\theta}+\delta$ for all $\hat{\theta}<\theta^{*}$ so the manager is paying out everything he says he has. When the manager reports $\hat{\theta}=-\delta$ and has $\theta \leq \theta^{*}$ he is paid $\bar{w}$. When we construct a "nice" contract in section 2.2 .3 , this is the route we will take.

While we have treated the beliefs of the monitors with respect to the reports not made in equilibrium; $\hat{\theta} \in\left(0, \theta^{*}\right)$; as a free variable, if $\bar{w}>\tau \phi$, we can construct a minor permutation of this arrangement that pins them down. Start first with the case in which $E\left\{\theta \mid \theta<\theta^{*}\right\}>\phi$. We construct the revised reporting strategy of the manager as follows. For each output $\theta \in\left(0, \theta^{*}\right)$ and $\tilde{\theta}=\min \left\{\theta^{\prime}: \theta^{\prime}>\theta+\phi\right\}$ we select the probabilities that $\theta$ tells the truth $\psi_{1}$ and the probability $\psi_{2}$ that type $\tilde{\theta}$ reports $\theta$ so that

$$
\frac{\psi_{2}}{\psi_{1}+\psi_{2}}[\tilde{\theta}-\phi]=0
$$

and $\psi_{1}+\psi_{2}=\psi$. So long as $\psi$ is sufficiently small, then none of our types is being asked to change the probability of his misreporting by an infeasible degree and the condition expectation of the monitors upon receiving a report of 0 is still high enough to motivate monitoring. However, the expected return to monitoring under the perturbed reports is exactly $\phi$ for $\hat{\theta} \in\left(0, \theta^{*}\right)$. The perturbed reporting strategy will lead to more monitoring. However, as $\psi$ goes to zero, the cost of this perturbation becomes arbitrarily small. Finally note that we will have to change the compensation schedule to make the manager indifferent between truthtelling and these perturbed reports. However since $\bar{w}>\tau \phi$, none of the reporting deviations are large enough to trigger a renegotiation of compensation.

Even if (14) is not satisfied, then can still apply the logic of this argument by simply increasing $\delta$ by $\varepsilon$. As $\psi$ goes to 0 , the required $\varepsilon$ will also go to zero. and the cost of the permutation will again get small.

Since without commitment outside investors cannot be compelled to make further investments in the firm, they will need to invest enough to insure that there are sufficient resources to cover the managers compensation and the costs of monitoring for all possible $\theta$. Given our compensation scheme, it follows that $\bar{w}+\delta$ must be invested up front. However since the $\bar{w}$ 
investment is done via long-term funds, investing it up front involves no additional costs. In contrast, the $\delta$ funds invested up front are costly since they are done via liquid funds.

\subsubsection{Randomization Increases Efficiency}

Here we want to illustrate how randomized monitoring can improve the efficiency of the contract. The reason that monitoring is taking place for all reports $\hat{\theta} \in\left[0, \theta^{*}\right)$ is to make $\theta^{*}$ high enough so as prevent managers with output levels $\theta>\theta^{*}$ from having an incentive to misreport $\theta^{*}$ in order to get $\tau\left(\theta-\theta^{*}\right)$ instead of $w(\theta)$. However, even for a misreport of 0 , it is not necessary to set the reporting probability to one in order to deter misreporting if $\bar{w}>0$.

This is easiest to see in the deterministic contract with commitment. Taking as given the wage contract $w(\theta)$, and the interim payment schedule (payout $\hat{\theta}$ if $\hat{\theta}<\theta^{*}$ and payout $\theta^{*}$ if $\hat{\theta} \geq \theta^{*}$, we can construct the minimal monitoring probability to support this contract recursively. Let's denote our set of outputs by $\Theta=\left\{\theta_{0}, \theta_{1}, \ldots, \theta_{N}\right\}$, where $\theta_{j}<\theta_{j+1}, \theta_{0}=0$, and $\theta_{N}$ is the upper support, and we take $\theta^{*}=\theta_{J}$. Then, $m\left(\theta_{j}\right)=0$ for all $j \geq J$. For each $j$ from $J-1$ to 0 we can construct the minimal monitoring level necessary to support $w\left(\theta^{*}\right)$ by the requirement that the probability of monitoring be just enough to deter the type who has the most to gain from misreporting, or

$$
m\left(\theta_{j}\right): \max _{i>j}\left[u\left(w\left(\theta_{i}\right)-u\left(\tau\left(\theta_{i}-\theta_{j}\right)\right)\left(1-m\left(\theta_{j}\right)\right)\right]=0 .\right.
$$

It's easy to see that the monitoring levels in this construction will be declining in $j$ since $\tau\left(\theta_{i}-\theta_{j}\right)$ is declining for each $i$, and even $m\left(\theta_{0}\right)<1$ if $\bar{w}>0$ since $w(\theta) \geq \bar{w}$. Hence, we have improved on the deterministic contract with commitment by lowering the monitoring costs associated with it. ${ }^{12}$

If we require that monitoring be self-enforcing, then constructing a stochastic monitoring contract that improves efficiency becomes more difficult. To illustrate how such a contract can be constructed, start from our deterministic self-enforcing contract for the case in which $E\left\{\theta \mid \theta<\theta^{*}\right\}>\phi$ (which implies that $\delta=0$ ) and $\bar{w} \geq \tau \phi$. Consider an alterative mechanism in which we partitioning the interval $\left\{\theta_{0}, \ldots, \theta_{J-1}\right\}$ into $\left\{\theta_{0}, \ldots, \theta_{I}\right\}$ and $\left\{\theta_{I+1}, \ldots, \theta_{J-1}\right\}$, where $E\left\{\theta \mid \theta \leq \theta_{I}\right\} \geq \phi$. Managers with output $\theta \leq \theta_{I}$ still report output of 0 and are monitored with probability 1 , and receive compensation $\bar{w}$. Managers with output $\theta \in\left\{\theta_{I+1}, \ldots, \theta_{J-1}\right\}$ report output $\theta-\phi$, and hence payout $\theta-\phi$ if they aren't monitored, and $\theta$ if they are monitored. (This may require expanding the type space $\Theta$ to include these probability

\footnotetext{
${ }^{12}$ Boyd and Smith (1994) have argued in an numerical example that the gains from stochastic monitoring are small. However this example suggests that they can be made quite large.
} 
zero types). Note that with this interim payout schedule, any monitor who receives one of these reports is by construction just indifferent between monitoring and not. Note also that since $\bar{w}>\tau \phi$, the types that make these misreports cannot renegotiate their wage contract upwards. We are then free to set the monitoring probabilities for these types so as to just prevent any higher output type from misreporting this output level. This will allow us to economize on the monitoring costs for all $\theta \in\left\{\theta_{I+1}, \ldots, \theta_{J-1}\right\}$.

If $\bar{w}<\tau \phi$ then these types that misreport downwards will be able to renegotiate their wage up to $\tau \phi$ if monitoring does not take place. In which case, the compensation schedule will have to be changed to keep them indifferent, or

$$
u(\tau \phi)(1-m(\theta-\phi))+u\left(x_{1}(\theta-\phi, \theta)\right) m(\theta-\phi)=u(\bar{w})
$$

and their consumption when they aren't monitored, $\tau \phi$, is higher than their consumption when they are monitored, $x_{1}(\theta-\phi, \theta)<\bar{w}$; though as $m(\theta-\phi) \rightarrow 1, x_{1}(\theta-\phi, \theta) \rightarrow \bar{w}$. This consumption gap is inefficient, and will imply that their expect level of compensation will be higher than before. As a result, there is now a cost associated with reducing the extent of monitoring coming from the fact that the equilibrium level of consumption is not independent of monitoring, and the net change in the payoff of the outside investors is given by

$$
\frac{d}{d m(\theta-\phi)}(1-m(\theta-\phi))[\tau \phi-\bar{w}-\gamma]+m(\theta-\phi)\left[x_{1}(\theta-\phi, \theta)-\bar{w}\right] .
$$

Note that making $\phi$ as small as possible helps to reduce the consumption distortions, and shrinks the necessary level of the bottom partition, $\theta \leq \theta_{I}$, where monitoring is taking place with probability one. ${ }^{13}$

\subsection{Stochastic Monitoring}

In this section we characterize the efficient contract with self-enforcing stochastic monitoring. We will first consider the case of stochastic monitoring with commitment.

\footnotetext{
${ }^{13}$ We have assumed that both output and liquid funds left in the firm between the second and third subperiods lose the fraction $\tau$ of their value if the manager quits at the beginning of the third subperiod. If however we assumed that liquid funds were not subject to a loss if the manager quits, and if the contract could ensure that the no-monitoring interim payment was first paid with output and then liquid funds, the gap between $v_{1}$ and $v_{0}$ would be largely (if not solely) composed of liquid funds, which would imply that there would be no wage renegotiation and the model would work like the case in which $\bar{w} \geq \tau \phi$. (See footnote 6 for further discussion.)
} 


\subsubsection{Commitment}

Just as in the deterministic case with commitment, here too the standard revelation principal applies, and we can restrict ourselves to a truthtelling equilibrium in which $r(\theta, \theta)=1$ and $r(\hat{\theta}, \theta)=0$ for all $\hat{\theta} \neq \theta$. The problem becomes one of maximizing (11) subject to the feasibility conditions (1-5), the renegotiation proof constraint on $x_{i}$, (6), the incentive constraint that truth-telling is a best response, (7). We have dropped both the best response constraint on other reports given with positive probability, (8), and the monitoring incentive constraint (10). We characterize the solution to this contracting problem in the following propositions.

Proposition 2.2. There is an efficient contract with the following properties:

1. $v_{1}(\hat{\theta}, \theta)=\theta$ and $v_{0}(\hat{\theta})=\hat{\theta}$ for all $\hat{\theta}<\theta^{*} \equiv \min \{\theta \in \Theta: m(\theta)=0\}$ and $v_{0}(\hat{\theta})=\theta^{*}$ for all $\theta \geq \theta^{*}$

2. $x_{1}(\hat{\theta}, \theta)=0$ and $x_{0}(\hat{\theta}, \theta)=\tau\left(\theta-v_{0}(\hat{\theta})\right)$ for $\theta \neq \hat{\theta}$,

3. $x_{0}(\theta, \theta)=x_{1}(\theta, \theta)$,

4. $m(\theta)=0$ for all $\theta \geq \theta^{*}$, and

5. $\delta=0$.

Proof: The proof of this proposition is essentially the same as in the deterministic case. To prove part (1), note that $v_{1}$ and $v_{0}$ only show up in the renegotiation constraint (6), and that increasing their values to the maximum extent allowed by the limited liability constraints (2) and (3) relaxes the renegotiation constraint. Hence, it is efficient to do so. However, for reports above $\theta^{*}$ no further relaxation is possible since the renegotiation constraint and incentive constraints can again be combined to form

$$
\max _{\tilde{\theta} \in\left[\theta^{*}, \theta\right) \cap \Theta}(1-m(\tilde{\theta})) u(\tau[\theta-\tilde{\theta}])=u\left(\tau\left[\theta-\theta^{*}\right]\right),
$$

and hence there is no further relaxation in the incentive constraint from higher payments in the second sub-period above the level of the monitoring threshold $\theta^{*}$. Given (1) and the fact that it is efficient to punish misreporting to the maximum feasible extent, since it will not occur in equilibrium, (2) follows. Given (1), it follows that under truthtelling the no-perks constraints cannot bind, and hence, fixing the expected utility provided to the manager in state $\theta$ at $y(\theta)$, where

$$
y(\theta)=m(\theta) u\left(x_{1}(\theta, \theta)\right)+(1-m(\theta)) u\left(x_{0}(\theta, \theta)\right),
$$


any efficient contract must minimize the cost of delivering this utility. In other words, it must solve the following sub-problem

$$
\min _{x_{0}, x_{1}} m(\theta) x_{1}+(1-m(\theta)) x_{0} \text { s.t. } m(\theta) u\left(x_{1}\right)+(1-m(\theta)) u\left(x_{0}\right) \geq y(\theta) .
$$

The concavity of $u$ implies that this minimization is achieved with $x_{1}=x_{0}$. Finally, given that $\theta^{*}$ determines the extent to which the incentive and renegotiation constraint binds, there is no further benefit from monitoring for reports $\hat{\theta}>\theta^{*}$, and, since monitoring is costly, it follows that it therefore occurs with probability zero. Q.E.D.

Given proposition 2.2, we can simplify the problem to be one of choosing $[m(\theta), w(\theta)]$ so as to

$$
\max \sum[\theta-w(\theta)-\gamma m(\theta)] p(\theta)
$$

subject to

$$
\begin{gathered}
\sum u(w(\theta)) p(\theta)=U_{0}, \\
u(w(\theta)) \geq \max _{\tilde{\theta}<\theta}(1-m(\tilde{\theta})) u(\tau[\theta-\tilde{\theta}]),
\end{gathered}
$$

where we use $w(\theta)$ to denote $x_{i}(\theta, \theta)$, and we have made use of (15) in (18).

Proposition 2.3. In any solution to the simplified problem:

( $i) w(\theta)$ is such that there exists a $\bar{\theta}>\theta^{*}$ such that $w(\theta)=\bar{w}$ for all $\theta \leq \bar{\theta}$, and strictly increasing thereafter,

(ii) $m(\theta)$ is weakly decreasing.

If $\bar{w}$ is strictly greater than 0 , then

(iii) $m(\theta)<1$ and is strictly decreasing in the interior, and

(iv) $\theta^{*}<\max (\Theta)$.

Proof: See the Appendix.

The results with stochastic and deterministic monitoring under commitment are very similar. The result that the stochastic monitoring schedule is strictly decreasing for all nonzero monitoring levels becomes, under deterministic monitoring the result that the monitoring is weakly decreasing, which implies that it is done on an interval of shock reports starting from the lowest level. With respect to compensation, the results are somewhat more stark with deterministic monitoring: the efficient contract is completely specified by the base pay of the manager, $\bar{w}$ and the upper support of the monitoring set $\theta^{*}$. The reason for this difference is that in the stochastic monitoring case the efficient contract optimally trades off increasing consumption and decreasing the extent of monitoring, which smooths the change in the consumption schedule relative to deterministic monitoring. 
There is one important difference with respect to the interim payment that we associate with debt between deterministic and stochastic monitoring. Stochastic monitoring means that despite not paying the face value of the debt, $\theta^{*}$, monitoring may not occur. We would interpret this outcome as one in which the debtors and the firm reached a settlement and the gap between the announced level of the debt and the face value, $\theta^{*}-\hat{\theta}$, is forgiven and the firm simply pays $\hat{\theta}$ to avoid monitoring.

\subsubsection{Self-Enforcing Monitoring}

Here, we construct a contracting problem to characterize the efficient self-enforcing contract taking $\phi$ as given. In this contracting Lagrangian, we denote the multipliers associated with the different constraints by $a_{i}$ for $i=1, \ldots, 10$, and let $\hat{\theta}$ denote the reported type. The problem is to maximize over the choice of $\left\{\delta, v_{0}(\hat{\theta}), v_{1}(\hat{\theta}, \theta), x_{i}(\hat{\theta}, \theta), m(\hat{\theta}), r(\hat{\theta}, \theta)\right\}$ for all $\hat{\theta}$ and $\theta$ in order to solve the following problem.

$$
\begin{aligned}
& L=\max \sum_{\theta} \sum_{\hat{\theta}}\left\{\theta-\left[m(\hat{\theta})\left[x_{1}(\hat{\theta}, \theta)+\gamma\right]+(1-m(\hat{\theta})) x_{0}(\hat{\theta}, \theta)\right] r(\hat{\theta}, \theta)\right\} p(\theta)-\beta \delta \\
& +a_{1} \delta+\sum_{\hat{\theta}} a_{2}(\hat{\theta})\left[\hat{\theta}+\delta-v_{0}(\hat{\theta})\right]+\sum_{\theta} \sum_{\hat{\theta}} a_{3}(\hat{\theta}, \theta)\left[\theta+\delta-v_{1}(\hat{\theta}, \theta)\right] \\
& +\sum_{\theta} \sum_{\hat{\theta}}\left\{a_{4}(\hat{\theta}, \theta)\left[x_{0}(\hat{\theta}, \theta)-\tau\left(\theta+\delta-v_{0}(\hat{\theta})\right)\right]\right\} \\
& +\sum_{\theta} \sum_{\hat{\theta}}\left\{a_{5}(\hat{\theta}, \theta)\left[x_{1}(\hat{\theta}, \theta)-\tau\left(\theta+\delta-v_{1}(\hat{\theta}, \theta)\right)\right]\right\} \\
& +\sum_{\theta} \sum_{\hat{\theta}} a_{6}(\hat{\theta})\left[\theta+\delta-v_{0}(\hat{\theta})\right] r(\hat{\theta}, \theta) \\
& \left.+\sum_{\theta} \sum_{\hat{\theta}}\left\{\begin{array}{c}
{\left[a_{7}(\hat{\theta}, \theta)-a_{8}(\hat{\theta}, \theta) r(\hat{\theta}, \theta)\right] \times} \\
m(\theta) u\left(x_{1}(\theta, \theta)\right)+(1-m(\theta)) u\left(x_{0}(\theta, \theta)\right) \\
-m(\hat{\theta}) u\left(x_{1}(\hat{\theta}, \theta)\right)-(1-m(\hat{\theta})) u\left(x_{0}(\hat{\theta}, \theta)\right)
\end{array}\right]\right\} \\
& +a_{9}\left\{\sum_{\theta} \sum_{\hat{\theta}}\left[m(\hat{\theta}) u\left(x_{1}(\hat{\theta}, \theta)\right)+(1-m(\hat{\theta})) u\left(x_{0}(\hat{\theta}, \theta)\right)\right] r(\hat{\theta}, \theta) p(\theta)-\bar{U}\right\} \\
& +\sum_{\hat{\theta}} a_{10}(\hat{\theta})\left\{\sum_{\theta}\left[v_{1}(\hat{\theta}, \theta)-v_{0}(\hat{\theta})-\phi\right] r(\hat{\theta}, \theta) p(\theta)\right\}
\end{aligned}
$$


The first constraint is the nonnegativity constraint on $\delta$, the next two constraints are the feasibility constraint on the interim payments, the following two constraints are the renegotiation proofness constraints on managerial consumption, the sixth constraint is the reporting feasibility constraint, while following constraint incorporates both the incentive constraint $\left(a_{7}(\hat{\theta}, \theta)\right)$ and the equal utility constraint $\left(a_{8}(\hat{\theta}, \theta)\right)$ for reports that are made with positive probability. The ninth constraint is the promised utility or participation constraint. The tenth and final constraint is the incentive constraint on the monitors. In addition, we will have the constraints that $m(\hat{\theta}) \in \triangle(\Theta)$ and $r(\hat{\theta}, \theta) \in \triangle(\Theta) \times \Theta$.

Next we show that the monitoring incentive constraint can only bind in one direction, from below. This has significant implications for the nature of the efficient contract. The proof works off the fact that we can construct an alternative mechanism which relaxes the incentive constraint on the monitors if the constraint bound from above. We will later use this same logic to prove a key aspect of our debt/equity characterization of the efficient contract.

Lemma 1: The monitoring incentive constraint can never bind from above and hence $a_{10}(\hat{\theta}) \geq 0$.

Proof: Consider the following augmentation of the mechanism. Assume that there is a report $\hat{\theta}$ for which there is a $\theta$ type for whom $v_{1}(\hat{\theta}, \theta)<\theta+\delta$ and $r(\hat{\theta}, \theta)>0$. There are three cases to consider:

Case 1: If $\theta+\delta>v_{0}(\hat{\theta})+\phi$, then we can simply shift some of its reporting probability to an alternative report $\omega$, where $v_{0}(\omega)=\theta+\delta-\phi, v_{1}(\omega, \theta)=\theta+\delta$, and $m(\omega)=m(\hat{\theta})$. This shifting will relax the incentive constraint on the monitor for report $\hat{\theta}$, which will in turn allow $v_{1}(\hat{\theta}, \theta)$ to be increased towards $\theta$ and thereby relax the renegotiation proofness constraint both for reports $\hat{\theta}$ and $\omega$ for type $\theta$.

Case 2: $v_{1}(\hat{\theta}, \theta)-v_{0}(\hat{\theta})=\phi$ for all $\theta$ types for whom $r(\hat{\theta}, \theta)>0$. Then we can proceed exactly as in Case 1, except this doesn't relax the monitoring incentive constraint for report $\hat{\theta}$. However, the relaxation of the renegotiation proofness constraint for the type who now makes the new report $\omega$ still occurs.

Case 3: $\theta+\delta \leq v_{0}(\hat{\theta})+\phi$. However, it follows that there must exist a type $\tilde{\theta}$ for whom $v_{1}(\hat{\theta}, \tilde{\theta})-v_{0}(\hat{\theta})>\phi$. By shifting some of the reporting probability of type $\tilde{\theta}$ to the new report $\omega$, and setting $v_{0}(\omega)=\tilde{\theta}+\delta-\phi, v_{1}(\omega, \tilde{\theta})=\tilde{\theta}+\delta$, and $m(\omega)=m(\hat{\theta})$ we relax the renegotiation constraint for this type and also the monitoring incentive constraint for reports $\hat{\theta}$, which allows us to increase $v_{1}(\hat{\theta}, \theta)$ towards $\theta$. (Note that case 1 and case 3 are essentially the same, except that in case $1, \tilde{\theta}=\theta$ and the type whose $v_{1}$ payment we are increasing is the same as the type we are shifting to the alternative message $\omega$.)

Hence, the monitoring incentive constraint never binds from above or we can improve 
upon this mechanism. However this contradicts BS's result that this mechanism can support the efficient outcome. In the next section we construct such an alternative mechanism in order to generate a "nice" contract. Q.E.D.

An implication of the one-sided binding of the monitor's incentive constraint is that the size of the incentive wedge should be made as small as possible and hence the complete separation of claims is efficient.

Proposition 2.4. It is efficient to set $\phi=\gamma$; thereby making $\phi$ as small as possible.

Proof: The derivative of the Lagrangian with respect to $\phi$ is given by

$$
\frac{\partial L}{\partial \phi}=-\phi \sum_{\hat{\theta}} a_{10}(\hat{\theta}) \sum_{\theta} r(\hat{\theta}, \theta) p(\theta) .
$$

Since we have already established that $a_{10}(\hat{\theta}) \geq 0$, it follows that if any of the incentive constraint on the monitors ever binds, it is efficient to make $\phi$ as small as possible. Hence, the complete separation of claims is always at least weakly efficient. Q.E.D.

We characterize the optimal contract in more detail in the appendix. However, the key additional point we wish to establish is the following.

Proposition 2.5. Let $\theta^{*}=\min \{\hat{\theta}: m(\hat{\theta})=0\}$. It is efficient to set $v_{0}(\hat{\theta})=\theta^{*}+\delta$ if $\hat{\theta}$ is s.t. $m(\hat{\theta})=0$, and to set $m(\tilde{\theta})=0$ if $v_{0}(\tilde{\theta}) \geq \theta^{*}+\delta$.

Proof: Just as in the commitment case, it follows from the incentive constraint that if $m(\theta)=0$, then

$$
x_{0}(\theta, \theta) \geq \tau\left(\theta+\delta-\min _{\hat{\theta} \in\{\theta: m(\theta)=0\}} v_{0}(\hat{\theta})\right) .
$$

Hence, there is no gain from raising $v_{0}(\theta)$ above the minimum interim payment from reports that do not lead to monitoring. There is a gain to making this minimum payment as large as possible and hence equal to $\theta^{*}+\delta$. Moreover, since in this case we can set $v_{1}(\theta, \tilde{\theta})=v_{0}(\theta)$ there is no problem satisfying the incentive constraint on the monitors.

To see that there is no gain to monitoring for reports that lead to a no-monitoring interim payment $v_{0}(\tilde{\theta}) \geq \theta^{*}+\delta$, note that monitoring at $\tilde{\theta}$ doesn't relax any types incentive constraint. Then, note that since every type $\tilde{\theta}>\theta^{*}$ must receive a payoff of at least $u\left(\tau\left(\tilde{\theta}-\theta^{*}\right)\right)$, and the cheapest way to deliver a given payoff $y$ is to set consumption equal to $u^{-1}(y(\theta))$ and not monitor. This result also is very similar to what we saw with commitment among reports that didn't lead to monitoring. Q.E.D. 


\subsubsection{Nice Contract}

In this section we show that the efficient arrangement can be supported with a contract in which the interim payment resembles debt and the final payment resembles equity. We will say that the efficient contract resembles debt and equity if the following properties hold:

A. for reports that can trigger monitoring, everything is taken, or $v_{1}(\hat{\theta}, \theta)=\theta+\delta$ and $v_{0}(\hat{\theta})=\hat{\theta}+\delta$ if $m(\hat{\theta})>0 .{ }^{14}$

B. for high enough reports that do not trigger monitoring, a constant amount is taken which is weakly larger than that what can trigger monitoring, or $v_{0}(\hat{\theta})=\theta^{*}+\delta \geq$ $\max \{\hat{\theta}: m(\hat{\theta})>0\}+\delta$.

We will construct this alternative solution through a series of propositions. In doing so, we will need to use a message space that is larger than the type space. This message space will include both the manager's type and a suggested monitoring probability, and the manager's type will include more types than just those in $\Theta$.

Note that we have already established the following properties: (i) $v_{1}(\hat{\theta}, \theta)=\theta+\delta$ or it doesn't effect the solution, (ii) if we order reports by their implied no-monitoring interim payments $v_{0}(\hat{\theta})$, then there is a cutoff interim payment such that monitoring only occurs for payments below a certain threshold and doesn't for payments above that threshold, and (iii) that it is efficient to have this threshold given by $\theta^{*}+\delta$, where $\theta^{*}$ is the smallest output report for which there is not monitoring. So all that we need to do here is to establish property A.

In the next proposition we will construct an alternative mechanism in which we always take everything that the manager says he has if we monitor with positive probability. Just as in the deterministic case, this will involve allowing for negative output reports.

To understand this proposition, consider the following simple example. Consider two output types, $\theta=10$ and $\theta=15$, and assume that their reported outputs, monitoring levels and interim payment if not monitored were as given in the table labeled original mechanism. Assume for simplicity that $\delta=0$. We want to construct a new mechanism in which both of these types report as their output the amount of their interim payment if not monitored. However, this amount is the same despite the fact that they are being monitored with different probabilities. So, to achieve the same outcome as before, under the new mechanism we simply have them report both the amount of their interim payment if not monitored and a suggested monitoring probability. Note that the scope of deviations is unchanged since

\footnotetext{
${ }^{14}$ Note that the liquid funds $\delta$ may not be paid out if monitoring doesn't occur in order to augment the incentive to monitor. Just as in the case with deterministic monitoring, if negative reports $\hat{\theta}=-\delta$ are allowed, then we get that $v_{0}(\hat{\theta})=\hat{\theta}+\delta$ if $m(\hat{\theta})>0$.
} 
lying about one's suggested monitoring probability is equivalent to reporting 10 instead of 7 under the original mechanism (or vice versa).

ORIGINAL MECHANISM
\begin{tabular}{|l|l|l|}
\hline$\theta$ & 10 & 15 \\
\hline$\omega$ & 7 & 10 \\
\hline$m$ & $1 / 2$ & $1 / 4$ \\
\hline$v_{0}$ & 5 & 5 \\
\hline
\end{tabular}

NEW MECHANISM
\begin{tabular}{|l|l|l|}
\hline$\theta$ & 10 & 15 \\
\hline$\omega$ & $(5,1 / 2)$ & $(5,1 / 4)$ \\
\hline$m$ & $1 / 2$ & $1 / 4$ \\
\hline$v_{0}$ & 5 & 5 \\
\hline
\end{tabular}

We will denote the new message space by $\Omega$ and a generic message by $\omega$. Let $\Omega=\tilde{\Theta} \times \tilde{\Pi}$ where

$$
\tilde{\Theta}=\Theta \cup\left\{\theta^{\prime}: v_{0}(\hat{\theta})-\delta=\theta^{\prime} \text { for some } \hat{\theta} \in \Theta\right\}
$$

and

$$
\tilde{\Pi}=\{\pi \in[0,1]: m(\hat{\theta})=\pi \text { for some } \hat{\theta} \in \Theta\} \cup\{0,1\}
$$

Consistent with our prior notation, a reporting strategy $r$ is a probability distribution over $\Omega$, which we denote by $r \in \triangle(\Omega) \times \Theta$, where $r(\omega, \theta)$ is the probability that a manager of type $\theta$ makes a report $\omega$. We will denote by $\hat{\theta}(\omega)$ the output level in the report $\omega$ and by $\hat{\pi}(\omega)$ the suggested monitoring level.

Proposition 2.6. Given any solution to this problem, one can construct an equivalent solution in which $v_{0}(\omega)=\hat{\theta}(\omega)+\delta$ if $m(\hat{\theta}(\omega))>0$.

Proof: See the Appendix.

Given this result, we will henceforth work with the alternative constructed solution in which the amount taken when there is no monitoring is equal to the reported output level, or $v_{0}(\omega)=\hat{\theta}(\omega)+\delta$, and managers not only report their output level but make a suggestion as to their monitoring probability. Note that in our constructed alternative solution, for all messages made with positive probability, $m(\omega)=\hat{\pi}(\omega)$. Also, $r(\omega, \theta)=0$ for all $\hat{\theta}(\omega)>\theta$; that is, managers only misreport down.

We next show that taking everything when monitoring occurs is efficient by constructing an alternative to our alternative solution in which this is the case. We have already shown 
that in the original contract if either the monitoring inventive constraint binds at report $\hat{\theta}$, or renegotiation proofness constraint binds for type $\theta$ making report $\hat{\theta}$, then $v_{1}(\hat{\theta}, \theta)=\theta+\delta$, and hence $v_{1}(\hat{\theta}, \theta)<\theta+\delta$ only if it doesn't effect the value of the solution. So this is a fairly limited result, and but it does make clear that the efficient arrangement can have this feature everywhere that monitoring takes place with positive probability.

The logic of the argument here is essentially the same as in Lemma 1. To understand what is happening it is useful to consider the following simple example. In this example, we will assume that $\phi=2.5$ and $\delta=0$. The example considers two types, $\theta=5$ and $\theta=15$ who are both reporting $\omega=(5,1 / 2)$ under the new mechanism constructed above. However, $v_{1}[(5,1 / 2), 15]=10$, which is less than the total output of this type. This interim payment in the case of monitoring cannot simply be adjusted up however, since doing so would raise the expected payoff from monitoring to be above $\phi=2.5$, and hence give the monitors an incentive to monitor with probability one. To prevent this, we spit this type into two groups, rasing the first groups interim payment and having the second group make a different report. This change is illustrated in the second table labeled new new mechanism. The key to note here is that both for the message $(5,1 / 2)$ and the message $(15-2.5,1 / 2)$, the expected payoff from monitoring is $\phi$ since $(.1667 * 10) /(.1667+.5)=2.5$ and that this division of $\theta=15$ group originally reporting $(5,1 / 2)$ is feasible since $.1667+.3333=.5$.

NEW MECHANISM
\begin{tabular}{|l|l|l|}
\hline$\theta$ & 5 & 15 \\
\hline$\omega$ & $(5,1 / 2)$ & $(5,1 / 2)$ \\
\hline$\#$ & $1 / 2$ & $1 / 2$ \\
\hline$v_{1}$ & 5 & 10 \\
\hline
\end{tabular}

NEW NEW MECHANISM

\begin{tabular}{|l|l|l|l|}
\hline$\theta$ & 5 & 15 & 15 \\
\hline$\omega$ & $(5,1 / 2)$ & $(5,1 / 2)$ & $(15-2.5,1 / 2)$ \\
\hline$\#$ & $1 / 2$ & .1667 & 0.3333 \\
\hline$v_{1}$ & 5 & 15 & 15 \\
\hline
\end{tabular}

Proposition 2.7. Given an alternative solution to this problem constructed along the lines of proposition 2.6, in which $v_{0}(\omega)=\hat{\theta}(\omega)+\delta$, there is an equivalent solution in which $v_{1}(\omega, \theta)=\theta+\delta$ for all $\omega$ s.t. $m(\omega)>0$.

Proof: See the Appendix. 


\section{Concluding Comments}

We have considered a simple model of a firm which hires a manager to produce output subject to a long-run agency friction and a short-run information friction. We have allowed for stochastic monitoring because of its ability to efficiently economize on the extent of monitoring needed to induce the correct incentives on reporting. However, because monitoring is ex post inefficient, we have required it to be self-enforcing. We have shown that an efficient contract with self-enforcing monitoring has many of the features of standard debt and equity claims. First, the intermediate payment has a debt like characteristic in which everything is taken when monitoring occurs, and that it is efficient to have a flat payment equal to the highest report that can trigger monitoring with positive probability for reports so high that monitoring will not occur. However, unlike the case with deterministic monitoring, stochastic monitoring by its very nature features debt forgiveness as one of its primary features; that is, the failure to pay the face value of the debt does not automatically trigger monitoring. Also, unlike the commitment case, misreporting plays an important role in sustaining monitoring. It is only the expectation of misreports of lower output levels by the manager that induces the monitors to monitor since under the efficient contract all of the reported output is collected if it is below a threshold level. Finally, we have shown that complete separation of claims is efficient when monitoring is self-enforcing. This provides a rationale for the unbundling of the debt and equity payments coming out of the firm.

While we have generated these results within a one period model, it is relatively straightforward to show that a dynamic version of this model will also have these features. AC showed that their model, in which there was commitment by the principal and deterministic monitoring, preserved the debt and payment features described here when one considered an infinitely repeated version of their environment. The key to their result was that their information friction is temporary, as it is here.

\section{References}

[1] Albuquerque, R. and Hopenhayn, H. "Optimal Lending Contracts and Firm Dynamics", with Hugo Hopenhayn, September 2002. Forthcoming Review of Economic Studies.

[2] Aiyagari, S. Rao, and Fernando Alvarez. "Stationary Efficient Distributions With Private Information and Monitoring: A Tale of Kings and Slaves." Manuscript, Federal Reserve Bank of Minneapolis, 1995.

[3] Atkeson, A. and H. Cole. 2005. A Dynamic Theory of Optimal Capital Structure and 
Executive Compensation. NBER working paper \#11083.

[4] Bester, H. and R. Strausz. 2001. Contracting with Imperfect Commitment and the Revelation Principal: The Single Agent Case. Econometrica, vol. 69, No. 4.

[5] Berglöf, E. and E. von Thadden, 1994. "Short-Term Versus Long-Term Interests: Capital Structure with Multiple Investors," The Quarterly Journal of Economics, Vol. 109, No. 4 (Nov., 1994), pp. 1055-1084

[6] Border, K. and J. Sobel. 1987. Samurai Accountant: A Theory of Auditing and Plunder. Review of Economic Studies, vol.4, 525-540.

[7] Boyd, J. and B. Smith. 1994. How Good Are Standard Debt Contracts? Stochastic versus Nonstochastic Monitoring in a Costly State Verification Environment, Journal of Business, vol. 67: 530-561.

[8] Clementi, G.L. and Hopenhayn, H., "A Theory of Financing Constraints and Firm Dynamics," RCER working paper \#492, June 2002.

[9] DeMarzo, P. and Fishman, M., "Optimal Long-Term Financial Contracting with Privately Observed Cash Flows," Graduate School of Business, Stanford University, 2003.

[10] Dewatripont, M. and J. Tirole 1994. "A Theory of Debt and Equity: Diversity of Securities and Manager-Shareholder Congruence," The Quarterly Journal of Economics, Vol. 109, No. 4 (Nov., 1994).

[11] Gale, D. and M. Hellwig. 1985. Incentive-compatible Debt Contracts, Review of Economic Studies, vol. 52: 647-663.

[12] Hart, O. and J. Moore. 1995. Debt and Seniority: An Analysis of the Role of Hard Claims in Constraining Management, American Economic Review, 85(3): 567-85.

[13] Hart, O. and J. Moore, 1998. Default and Renegotiation: A Dynamic Model of Debt, Quarterly Journal of Economics, 113(1), 1-41.

[14] Jensen, M. 1986. Agency Costs of Free Cash Flow, Corporate Finance, and Takeovers, American Economic Review, 76(2): 323-29.

[15] Kedia, S. and T. Philippon. 2007. The Economics of Fraudulent Accounting, research memo NYU Stern School.

[16] Khalil, F. 1997. Auditing without Commitment, RAND Journal of Economics, vol. 28, 629-640. 
[17] Monnet, C. and E. Quintin. 2005. Optimal Contracts in a Dynamic Costly State Verification Model, Economic Theory, vol. 26: 867-885.

[18] Maskin, E. and J. Tirole. 1999. Unforeseen Contingencies and Incomplete Contracts, Review of Economic Studies, vol. 66, 83-114.

[19] Mookerjee, D. and I. Png. 1989. Optimal Auditing, Insurance and Redistribution, Quarterly Journal of Economics, vol. 104: 399-415.

[20] Townsend, R. 1979. Optimal Contracts and Competitive Markets with Costly State Verification, Journal of Economic Theory, 21(2): 265-93.

[21] Wang, C. , 2004. Dynamic Costly State Verification, research memo.

[22] Williamson, S. 1987. Costly Monitoring, Loan Contracts and Equilibrium Credit Rationing, Quarterly Journal of Economics, vol. 102: 135-145.

\section{Appendix}

Proof of Proposition 2.3: We first form the Lagrangian, which we formulate in terms of choosing the monitoring probabilities $m(\theta)$ and the utility level of the manager $y(\theta)$ to yield a convex constraint set.

$$
L=\sum_{\theta}\left\{\begin{array}{c}
{[\theta-C(y(\theta))-\gamma m(\theta)]+\lambda\left[y(\theta)-U_{0}\right]} \\
\sum_{\tilde{\theta}=0}^{\theta} \delta(\theta, \tilde{\theta})[y(\theta)-(1-m(\tilde{\theta})) u(\tau[\theta-\tilde{\theta}])] p(\tilde{\theta}) \\
\left.+\chi^{+}(\theta)(1-m(\theta))+\chi^{+}(\theta) m(\theta)\right)
\end{array}\right\} p(\theta)
$$

where $C(x)=u^{-1}(x), \lambda$ is the multiplier on the promise keeping constraint, $\delta(\theta, \tilde{\theta})$ is the multiplier on the incentive constraint (18) with respect to the deviation of reporting $\tilde{\theta}$ given a realization $\theta$, and $\chi^{+}(\theta)$ and $\chi^{+}(\theta)$ are the multipliers on the zero and one bounds on $m(\theta)$. The first-order conditions for this problem are

$$
\begin{gathered}
C^{\prime}(y(\theta))=\lambda+\sum \delta(\theta, \tilde{\theta}) p(\tilde{\theta}) \\
\gamma=\sum \delta\left(\theta^{\prime}, \theta\right) u\left(\tau\left(\theta^{\prime}-\theta\right)\right) p\left(\theta^{\prime}\right)-\chi^{+}(\theta)+\chi^{-}(\theta) .
\end{gathered}
$$

Condition (20) implies that if constraint (18) doesn't bind, then $y(\theta)=\bar{u}$, and moreover that $y(\theta) \geq \bar{u}$ for all $\theta$. In terms of the compensation of the manager, this implies that $w(\theta)=\bar{w}$ when the incentive constraint doesn't bind, where $\bar{w}=C(\bar{u})$. To see that if the constraint binds at $\theta_{1}$ and if $\theta_{2}>\theta_{1}$, then it binds at $\theta_{2}$, and moreover that $w\left(\theta_{1}\right)<w\left(\theta_{2}\right)$, note that 
$(1-m(\tilde{\theta})) u(\tau[\theta-\tilde{\theta}])$ is strictly increasing in $\theta$, which implies that $y\left(\theta_{2}\right)>y\left(\theta_{1}\right)$, and hence

$$
w\left(\theta_{2}\right)=C\left(y\left(\theta_{2}\right)\right)>C\left(y\left(\theta_{1}\right)\right)=w\left(\theta_{1}\right)
$$

which this proves (1).

To prove (2), note that condition (21) implies that if $m(\theta)>0$, then it must be the case that $\delta(\tilde{\theta}, \theta)>0$ for some $\tilde{\theta}>\theta$, or in other words that deviating and reporting $\theta$ binds on some type $\tilde{\theta}$. This result implies that if we take $y(\theta)$ as given, and define for each $\theta$ the probabilities of monitoring such that deviating is not weakly preferred, $\pi^{\theta}(\tilde{\theta})$, as follows

$$
\pi^{\theta}(\tilde{\theta})=\left\{\begin{array}{c}
\max \left\{0,1-\frac{y(\theta)}{u(\tau[\theta-\tilde{\theta}])}\right\} \text { if } \tilde{\theta}<\theta \\
0 \quad \text { o.w. }
\end{array},\right.
$$

then, efficiency implies that $m(\theta)=\max _{\tilde{\theta}}\left\{\pi^{\theta}(\tilde{\theta})\right\}$. In other words, the probability of monitoring is positive only if it binds for some type. (Note that by filling in the required probabilities with zeros we removed the need to shrink the type space that could deviate to $\theta$ as $\theta$ increased.) The function $\pi^{\theta}(\tilde{\theta})$ is weakly decreasing in $\tilde{\theta}$ for fixed $\theta$, and the sup over a set of weakly decreasing functions is weakly decreasing.

To prove (3), note first that $y(\theta) \geq \bar{u}=u(\bar{w})>0$. Then note that $(1-m(\theta)) u(\tau[\theta-\tilde{\theta}]) \leq$ $(1-m(\theta)) u(\tau(\sup (\theta))$, and that since $u(\tau(\max (\theta))$ is finite, the rhs goes to zero as $m(\theta)$ goes to one. Hence, $m(\theta)<1$ for all $\theta$.

To prove that $m$ is strictly decreasing in the interior when $\theta$ has finite upper support, assume that the reverse was true. That is, assume that for $\theta \in\left[\theta_{1}, \theta_{2}\right], m(\theta)=a$, where $0<a<1$ and $\theta_{2}-\theta_{1}>0$. Then, note that since for all finite $\tilde{\theta}>\theta_{1}$,

$$
(1-a) u\left(\tau\left[\tilde{\theta}-\theta_{1}\right]\right)>(1-a) u\left(\tau\left[\tilde{\theta}-\min \left(\theta_{2}, \theta\right)\right]\right),
$$

which implies that $\tilde{\theta}$ strictly prefers to report $\min \left(\theta_{2}, \theta\right)$ over $\theta_{1}$, and this contracts our earlier result that monitoring is only positive if it binds for some type, since monitoring is positive at $\theta_{1}$ and it does not bind there.

To prove $(i v)$, note that the incentive constraint (18) implies that if $\bar{w}>\tau(\theta-\hat{\theta})$, then deviating and making a report of $\hat{\theta}$ can only lower the payoff of the manager. Hence, for all $\theta$ such that $\tau(\sup (\Theta)-\theta)<\bar{w}$, monitoring is unnecessary and will optimally be set to zero. Note that since $w(\theta) \geq \bar{w}$, the interval over which $m(\theta)=0$, may be substantially larger than this simple bound would imply. Q.E.D.

\section{Discussion of first-order conditions:}


Here we characterize the efficient contract using the first-order conditions. The first-order condition for $\delta$ is given by

$$
-\beta+a_{1}+\sum_{\hat{\theta}} a_{2}(\hat{\theta})+\sum_{\theta} \sum_{\hat{\theta}}\left[\begin{array}{c}
a_{3}(\hat{\theta}, \theta)+a_{6}(\hat{\theta}) r(\hat{\theta}, \theta) \\
-\tau a_{4}(\hat{\theta}, \theta)-\tau a_{5}(\hat{\theta}, \theta)
\end{array}\right]=0
$$

The first-order conditions for $v_{0}(\hat{\theta})$ and $v_{1}(\hat{\theta}, \theta)$ are respectively given by

$$
-a_{2}(\hat{\theta})+\sum_{\theta}\left\{\tau a_{4}(\hat{\theta}, \theta)-a_{10}(\hat{\theta}) r(\hat{\theta}, \theta) p(\theta)-a_{6}(\hat{\theta}) r(\hat{\theta}, \theta)\right\}=0
$$

and

$$
-a_{3}(\hat{\theta}, \theta)+\tau a_{5}(\hat{\theta}, \theta)+a_{10}(\hat{\theta}) r(\hat{\theta}, \theta) p(\theta)=0 .
$$

To understand these conditions, note first that putting in liquid funds is costly and hence $\delta>0$ implies that the limited liability constraints on the size of $v_{0}(\hat{\theta})$ and $v_{1}(\hat{\theta}, \theta)$, and the reporting constraint bind sufficiently. Then, note that if the limited liability constraint on $v_{0}$ binds, then $a_{2}(\hat{\theta})>0$ and $v_{0}(\hat{\theta})=\hat{\theta}+\delta$. Similarly if the limited liability constraint on $v_{2}$ binds, then $a_{3}(\hat{\theta}, \theta)>0$ and $v_{1}(\hat{\theta}, \theta)=\theta+\delta$. If the renegotiations constraints bind $\left(a_{4}(\hat{\theta}, \theta)\right.$ and $\left.a_{5}(\hat{\theta}, \theta)\right)$ this promotes $v_{0}(\hat{\theta})=\hat{\theta}+\delta$ and $v_{1}(\hat{\theta}, \theta)=\theta+\delta$ respectively. If $r(\hat{\theta}, \theta)=0$, it is always efficient to set $v_{1}(\hat{\theta}, \theta)=\theta+\delta$. Similarly, if the sum over $\hat{\theta}$ of $r(\hat{\theta}, \theta) p(\theta)$ is equal to zero (the report $\hat{\theta}$ is not made with positive probability) it is efficient to set $v_{0}(\hat{\theta})=\hat{\theta}+\delta$. One factor weighting against setting $v_{0}(\hat{\theta})=\hat{\theta}+\delta$ and $v_{1}(\hat{\theta}, \theta)=\theta+\delta$ is the incentive constraint on the monitors and its multiplier $a_{10}(\hat{\theta})$. If the monitoring incentive binds and $a_{10}(\hat{\theta})>0$, it follows that $a_{3}(\hat{\theta}, \theta)>0$ (in which case $a_{5}(\hat{\theta}, \theta)=0$ ) and $v_{1}(\hat{\theta}, \theta)=\theta+\delta$. If $a_{10}(\hat{\theta})=0$ and the renegotiation proofness constraints $a_{5}(\hat{\theta}, \theta)$ doesn't bind, only then may it be the case that $v_{1}(\hat{\theta}, \theta)<\theta+\delta$. So, we get that either $v_{1}(\hat{\theta}, \theta)=\theta+\delta$ or it doesn't matter for the solution.

For $v_{0}(\hat{\theta})$, if $a_{10}(\hat{\theta})>0$ and $r(\hat{\theta}, \theta) p(\theta)>0$ for some $(\hat{\theta}, \theta)$, it follows that the renegotiation proofness constraint must bind for some type making the report of $\hat{\theta}$ with positive probability, and hence for this type $v_{0}(\hat{\theta})<\theta+\delta$.

We turn next to characterizing consumption for the manager. We will assume that the utility constraint binds and hence $a_{9}>0$. Define $\bar{w}$ by $u^{\prime}(\bar{w})=1 / a_{9}$. Then, note that the first-order conditions on $x_{0}(\theta, \theta)$ and $x_{1}(\theta, \theta)$ (that is, when the agent tells the truth) are 
respectively given by

$$
\begin{aligned}
0= & {\left[a_{9} u^{\prime}\left(x_{0}(\theta, \theta)\right)-1\right](1-m(\theta)) r(\theta, \theta) p(\theta)+a_{4}(\theta, \theta) } \\
& +(1-m(\theta)) u^{\prime}\left(x_{0}(\theta, \theta)\right)\left\{\sum_{\theta} \sum_{\hat{\theta}}\left[a_{7}(\hat{\theta}, \theta)-a_{8}(\hat{\theta}, \theta) r(\hat{\theta}, \theta)\right]\right\},
\end{aligned}
$$

and

$$
\begin{aligned}
0= & {\left[a_{9} u^{\prime}\left(x_{1}(\theta, \theta)\right)-1\right] m(\theta) r(\theta, \theta) p(\theta) } \\
& +m(\theta) u^{\prime}\left(x_{1}(\theta, \theta)\right)\left\{\sum_{\theta} \sum_{\hat{\theta}}\left[a_{7}(\hat{\theta}, \theta)-a_{8}(\hat{\theta}, \theta) r(\hat{\theta}, \theta)\right]\right\} .
\end{aligned}
$$

With commitment, the renegotiation proofness constraint could never bind on the efficient contract for truthful reports, else one could simply raise $v_{0}(\theta)$ and $v_{1}(\theta, \theta)$ to relax these constraints. Here it can bind with respect to $x_{0}(\theta, \theta)$, because the incentive constraint on the monitors could prevent $v_{0}(\theta)$ from being raised. If the incentive, best response and renegotiation proof constraints don't bind, then $x_{i}(\theta, \theta)=\bar{w}$, just as under commitment, and if they do bind, then $x_{i}(\theta, \theta)>\bar{w}$. Note that for the lowest type, $\theta=0$, the renegotiation constraint can never bind, and hence $w(0,0)=\bar{w}$.

The first-order conditions for managerial consumption under misreporting $(\hat{\theta} \neq \theta)$ are given by

$$
\begin{aligned}
0= & {\left[a_{9} u^{\prime}\left(x_{0}(\hat{\theta}, \theta)\right)-1\right](1-m(\hat{\theta})) r(\hat{\theta}, \theta) p(\theta)+a_{4}(\hat{\theta}, \theta) } \\
& -(1-m(\hat{\theta})) u^{\prime}\left(x_{0}(\hat{\theta}, \theta)\right)\left\{\sum_{\theta} \sum_{\hat{\theta}}\left[a_{7}(\hat{\theta}, \theta)-a_{8}(\hat{\theta}, \theta) r(\hat{\theta}, \theta)\right]\right\},
\end{aligned}
$$

and

$$
\begin{aligned}
0= & {\left[a_{9} u^{\prime}\left(x_{1}(\hat{\theta}, \theta)\right)-1\right] m(\hat{\theta}) r(\hat{\theta}, \theta) p(\theta) } \\
& -m(\hat{\theta}) u^{\prime}\left(x_{1}(\hat{\theta}, \theta)\right)\left\{\sum_{\theta} \sum_{\hat{\theta}}\left[a_{7}(\hat{\theta}, \theta)-a_{8}(\hat{\theta}, \theta) r(\hat{\theta}, \theta)\right]\right\} .
\end{aligned}
$$

For the case of misreports, the incentive constraint can bind, i.e. $a_{7}(\hat{\theta}, \theta)>0$, and this factor promotes setting $x_{0}(\hat{\theta}, \theta)$ and $x_{1}(\hat{\theta}, \theta)$ as low as possible. The best response condition binds in the opposite direction and can force up $x_{0}(\hat{\theta}, \theta)$ and $x_{1}(\hat{\theta}, \theta)$ so that the manager's 
payoff is equal to that from reporting the truth. Note that if $r(\hat{\theta}, \theta)=0$, then just as in the commitment case, it is efficient to set $x_{1}(\hat{\theta}, \theta)=0$, and $x_{0}(\hat{\theta}, \theta)$ as low as possible.

The first-order condition for monitoring on report $\hat{\theta}$ is given by

$$
\begin{aligned}
0= & \sum_{\theta}\left[x_{0}(\hat{\theta}, \theta)-x_{1}(\hat{\theta}, \theta)-\gamma\right] r(\hat{\theta}, \theta) p(\theta) \\
& -\left[a_{7}(\hat{\theta}, \theta)-a_{8}(\hat{\theta}, \theta) r(\hat{\theta}, \theta)\right]\left[u\left(x_{0}(\hat{\theta}, \hat{\theta})-u\left(x_{1}(\hat{\theta}, \hat{\theta})\right)\right]\right. \\
& +\sum_{\theta}\left\{\begin{array}{l}
{\left[a_{7}(\hat{\theta}, \theta)-a_{8}(\hat{\theta}, \theta) r(\hat{\theta}, \theta)\right] \times} \\
{\left[u\left(x_{0}(\hat{\theta}, \theta)\right)-u\left(x_{1}(\hat{\theta}, \theta)\right)\right]}
\end{array}\right\} \\
& -a_{9} \sum_{\theta}\left[u\left(x_{0}(\hat{\theta}, \theta)\right)-u\left(x_{1}(\hat{\theta}, \theta)\right)\right] r(\hat{\theta}, \theta) p(\theta) .
\end{aligned}
$$

We're being a bit loose here since we should also include multipliers on the constraints that $1 \geq m(\hat{\theta}) \geq 0$ and sum to one. If, as in the commitment case, $x_{0}(\hat{\theta}, \theta)=x_{1}(\hat{\theta}, \theta)$ for all reports made with positive probability $(r(\hat{\theta}, \theta)>0)$, we get that

$$
0=-\gamma \sum_{\theta} r(\hat{\theta}, \theta) p(\theta)+\sum_{\theta} a_{7}(\hat{\theta}, \theta) u\left(x_{0}(\hat{\theta}, \theta)\right)
$$

where we've made use of our result that $x_{1}(\hat{\theta}, \theta)=0$ if $r(\hat{\theta}, \theta)=0$ (and $u(0)=0$ by assumption). Just as in the commitment case, this expression implies that optimal monitoring is being chosen to dissuade misreporting, and for interior monitoring choices $m(\hat{\theta})$ is set just low enough to discourage misreporting. Moreover, the type that binds is the one in which $x_{0}(\hat{\theta}, \theta)$ is the largest, which is the highest $\theta$ type who is not suppose to make the report $\hat{\theta}$ since $x_{0}(\hat{\theta}, \theta)=\tau\left(\theta-v_{0}(\hat{\theta})\right)$.

However, if there is some $\theta$ type who is being asked to misreport his type as $\hat{\theta}$ with positive probability and for whom $x_{0}(\hat{\theta}, \theta) \neq x_{1}(\hat{\theta}, \theta)$, then monitoring also effects the degree to which the manager's consumption is being distorted. Note that since $v_{1}(\hat{\theta}, \theta)=\theta$ in this case, it follows that $x_{0}(\hat{\theta}, \theta)>x_{1}(\hat{\theta}, \theta)$ since the wage renegotiation condition can only bind when there is no monitoring.

The derivative of the objective function w.r.t. the reporting strategy $r(\hat{\theta}, \theta)$ is given by

$$
\begin{gathered}
\frac{\partial L}{\partial r(\hat{\theta}, \theta)}=-\left\{m(\hat{\theta})\left[x_{1}(\hat{\theta}, \theta)+\gamma\right]+(1-m(\hat{\theta})) x_{0}(\hat{\theta}, \theta)\right\} p(\theta) \\
-a_{8}(\hat{\theta}, \theta)\left[\begin{array}{c}
m(\theta) u\left(x_{1}(\theta, \theta)\right)+(1-m(\theta)) u\left(x_{0}(\theta, \theta)\right) \\
-m(\hat{\theta}) u\left(x_{1}(\hat{\theta}, \theta)\right)-(1-m(\hat{\theta})) u\left(x_{0}(\hat{\theta}, \theta)\right)
\end{array}\right]
\end{gathered}
$$




$$
\begin{aligned}
& +a_{9}\left[m(\hat{\theta}) u\left(x_{1}(\hat{\theta}, \theta)\right)+(1-m(\hat{\theta})) u\left(x_{0}(\hat{\theta}, \theta)\right)\right] p(\theta) \\
& +a_{10}(\hat{\theta})\left[v_{1}(\hat{\theta}, \theta)-v_{0}(\hat{\theta})-\phi\right] p(\theta) \partial \theta+a_{11}(\hat{\theta}, \theta)
\end{aligned}
$$

where we have added the multiplier $a_{11}(\hat{\theta}, \theta)$ to take account of the additional condition that $r(\hat{\theta}, \theta)$ sum to one over $\hat{\theta}$ 's. We have also made use of the fact that since the multiplier on the reporting constraint can only bind if the constraint holds, it follows that

$$
a_{6}(\hat{\theta}, \theta)\left[\theta+\delta-v_{0}(\hat{\theta})\right]=0
$$

This derivative may be positive or negative if $r(\hat{\theta}, \theta)$ is equal to one or zero respectively, and is equal to zero if it is interior.

Next, note that if the wage renegotiation constraints don't bind at $(\hat{\theta}, \theta)$ and $(\theta, \theta)$ $x_{i}(\hat{\theta}, \theta)=x_{0}(\theta, \theta)$ for $i=0,1$ and $x_{0}(\theta, \theta)=x_{1}(\theta, \theta)$. In this case, misreporting is only costly to the extent that $m(\hat{\theta})>m(\theta)$, and is beneficial to the extent that $a_{8}(\hat{\theta})>0$ and $\theta>\hat{\theta}$. However, the bigger the misreport in the sense of $\theta>\hat{\theta}$, the greater the extent to which the wage renegotiation constraint will bind when monitoring doesn't occur since $v_{0}(\hat{\theta})$ is constrained to be less than $\hat{\theta}$. In this case, misreporting has the additional costs of distorting consumption.

Consider reports which don't lead to monitoring, $m(\hat{\theta})=0$, in which case, the derivative with respect to the reporting probability simplifies to

$$
\begin{gathered}
\frac{\partial L}{\partial r(\hat{\theta}, \theta)}=-x_{0}(\hat{\theta}, \theta) p(\theta)-a_{8}(\hat{\theta}, \theta)\left[u\left(x_{0}(\theta, \theta)\right)-u\left(x_{0}(\hat{\theta}, \theta)\right)\right] \\
\left.+a_{9}[m(\hat{\theta})) u\left(x_{0}(\hat{\theta}, \theta)\right)\right] p(\theta)+a_{10}(\hat{\theta})\left[v_{1}(\hat{\theta}, \theta)-v_{0}(\hat{\theta})-\phi\right] p(\theta) \partial \theta+a_{11}(\hat{\theta}, \theta) .
\end{gathered}
$$

If $m(\theta)=0$ as well, then $x_{0}(\theta, \theta)=x_{0}(\hat{\theta}, \theta)$ from the combined implications of the incentive and the equal utility constraints. It then follows that since there are no gains from misreporting at $\hat{\theta}$ with positive probability, it is efficient to set $v_{1}(\hat{\theta}, \theta)=v_{0}(\hat{\theta})$ and thereby satisfy the monitoring incentive constraint (which implies that $a_{10}(\hat{\theta})=0$ ).

Proof of Proposition 2.6: Starting from any solution to the contracting problem above, we can construct an alternative mechanism $\left\{\tilde{\Omega}, \tilde{v}_{i}, \tilde{x}_{i}, \tilde{m}, \tilde{r}\right\}$ which supports the same outcome and in which

$$
\tilde{v}_{0}\left(\left[v_{0}(\hat{\theta})-\delta, m(\hat{\theta})\right]\right)=v_{0}(\hat{\theta})
$$

To do this we treat a manager who has reported $\left[v_{0}(\hat{\theta})-\delta, m(\hat{\theta})\right]$ as if he had reported $\hat{\theta}$. 
We do this first by setting

$$
\tilde{v}_{0}(\omega)=\hat{\theta}(\omega)+\delta \forall \omega \in \tilde{\Omega}
$$

Then, we require that for all $\hat{\theta} \in \Theta$,

$$
\begin{aligned}
\tilde{v}_{1}\left(\left[v_{0}(\hat{\theta})-\delta, m(\hat{\theta})\right], \theta\right) & =v_{1}(\hat{\theta}, \theta), \\
\tilde{m}\left(\left[v_{0}(\hat{\theta})-\delta, m(\hat{\theta})\right]\right) & =m(\hat{\theta}), \\
\tilde{x}_{i}\left(\left[v_{0}(\hat{\theta})-\delta, m(\hat{\theta})\right], \theta\right) & =x_{i}(\hat{\theta}, \theta),
\end{aligned}
$$

and that

$$
\tilde{r}\left(\left[v_{0}(\hat{\theta})-\delta, m(\hat{\theta})\right], \theta\right)=r(\hat{\theta}, \theta) .
$$

This determines all of the outcomes for all messages which are suppose to occur with positive probability; that is, for all

$$
\omega \in \vec{\Omega} \equiv\left\{\omega^{\prime} \in \tilde{\Omega}: v_{0}(\hat{\theta})-\delta=\hat{\theta}\left(\omega^{\prime}\right) \text { and } \hat{\pi}\left(\omega^{\prime}\right)=m(\hat{\theta}) \text { for some } \hat{\theta} \in \Theta\right\} .
$$

Note that if we restrict ourselves to $\omega \in \vec{\Omega}$, then in each of these outcomes, all of our conditions must be satisfied since they were satisfied in the original problem.

For all $\omega \notin \vec{\Omega}$, we need to ensure that no manager makes such a report, and, if $\hat{\theta}(\omega) \neq \theta$, that no manager will ever want to make such a report. To ensure that, we simply require that

$$
\begin{gathered}
\tilde{r}(\omega, \theta)=0, \\
\tilde{m}(\omega)=1, \\
\tilde{v}_{1}(\omega, \theta)=\theta+\delta,
\end{gathered}
$$

and

$$
\tilde{x}_{i}(\omega, \theta)=\left\{\begin{array}{c}
x(\theta, \theta) \text { if } \hat{\theta}(w)=\theta \\
0 \text { o.w. }
\end{array}\right.
$$

Note that for all $\omega \notin \vec{\Omega}$, no one is making these reports with positive probability and hence we are free to assign beliefs to the monitor so as to ensure any desired degree of monitoring. Since $\tilde{v}_{0}(\omega)=\hat{\theta}(\omega)+\delta$ and we set $\tilde{v}_{1}(\omega, \theta)=\theta+\delta$, we can set consumption as low as possible for anyone who reports $\omega \notin \vec{\Omega}$, and for whom the output report is not correct. There are many ways to set $\tilde{x}_{i}(\omega, \theta)$ since anything between 0 and the truth-telling level $x(\theta, \theta)$ will suffice. We have chosen somewhat arbitrarily to preserve the quality that telling 
the truth about one's output is a best response (irrespective of the recommended level of monitoring).Q.E.D.

Proof of Proposition 2.7: The basic idea of the proof is that if $v_{1}(\omega, \theta)<\theta+\delta$ and $\hat{\pi}(\omega)>0$, then we can take some of the managers who are reporting $\omega$ and for whom $\theta+\delta-v_{0}(\omega, \theta)>\phi$, and reassign them to report $[\theta-\phi, \hat{\pi}(\omega)]$. At this reassigned output level, we can set $v_{1}=\theta+\delta$ while $v_{0}=\theta+\delta-\phi$. Hence, $v_{1}-v_{0}=\phi$ and the monitoring self-enforcing constraint will still be satisfied with the inclusion of these types for a report of $[\theta-\phi, \hat{\pi}(\omega)]$. There is one special case that we have to deal with in which $v_{1}(\omega, \theta)-\hat{\theta}(\omega)=\phi$ for all the managers making the report of $\omega$ with positive probability. In what follows assume that $\hat{\pi}(\omega)>0$ for all the signals we consider.

First, consider the special case of an $\omega$ such that (i) for all $\theta$ such that $r(\omega, \theta)>0$, then $v_{1}(\omega, \theta)-\hat{\theta}(\omega)-\delta=\phi$, and (ii) for some $\theta^{\prime}: r\left(\omega, \theta^{\prime}\right)>0$, and $v_{1}\left(\omega, \theta^{\prime}\right)<\theta^{\prime}+\delta$. Then, we can simply have $\theta^{\prime}$ report $\left[\theta^{\prime}-\phi, \hat{\pi}(\omega)\right]$ and set $v_{1}\left(\left[\theta^{\prime}-\phi, \hat{\pi}(\omega)\right]\right)=\theta^{\prime}+\delta$. Note that this will not effect monitoring incentive constraint at either report $\omega$ or report $\left[\theta^{\prime}-\phi, \hat{\pi}(\omega)\right]$, and that this change in reporting relaxes the no-perks constraints on $x_{0}$ and $x_{1}$ but otherwise leaves the objective function unchanged, while satisfying all of the constraints. Hence, it must be weakly efficient.

Next, assume that there exists a $\theta^{\prime}$ such that $r\left(\omega, \theta^{\prime}\right)>0$, and $v_{1}\left(\omega, \theta^{\prime}\right)<\theta^{\prime}+\delta$ and a $\theta^{\prime \prime}: r\left(\omega, \theta^{\prime \prime}\right)>0$, and $v_{1}\left(\omega, \theta^{\prime \prime}\right)-\hat{\theta}(\omega)>\phi$. Note that it could be that $\theta^{\prime}=\theta^{\prime \prime}$. Then, raise $v_{1}\left(\omega, \theta^{\prime}\right)$ towards $\theta^{\prime}+\delta$, while offsetting the impact on the

$$
\sum_{\theta}\left\{\left[v_{1}(\omega, \theta)-\hat{\theta}(\omega)-\delta\right]-\phi\right\} r(\omega, \theta) p(\theta)
$$

by lowering $r(\omega, \theta$ ") and assigning the released reporting probability for type $\theta$ " by assigning it to report $[\theta "-\phi, \pi(\omega)]$, where $v_{1}(\theta "-\phi, \pi(\omega), \theta ")=\theta "+\delta$. Just as before, this change in reporting relaxes the no-perks constraint on $x_{1}\left(\omega, \theta^{\prime}\right), x_{1}\left(\omega, \theta^{\prime \prime}\right)$ and on $x_{0}\left(\omega, \theta^{\prime \prime}\right)$ but otherwise leaves the objective function unchanged, while satisfying all of the constraints including in particular the monitor incentive constraint. If the probability of type $\theta$ " reporting $\omega$ is exhausted before $v_{1}\left(\omega, \theta^{\prime}\right)$ is raised to $\theta^{\prime}$, simply find another $\theta^{\prime \prime}$ and continue the process.

Finally, note that if the incentive constraint could be satisfied under message $\omega$ with monitoring probability $m(\omega)$, then it can be satisfied for the message $(\theta "-\phi, \pi(\omega))$ since amount being paid out when monitoring does not occur is higher. and we are free to set the consumption level of types who are not suppose to make this report to zero when monitoring occurs. Hence, the incentive constraint holds strictly. Q.E.D. 